\title{
The ESCRT protein CHMP5 restrains skeletal progenitor cell senescence by preserving endo-lysosomal-mitochondrial network
}

Xianpeng $\mathrm{Ge}^{1^{*}}$, Lizhi $\mathrm{He}^{2}$, Haibo Liu ${ }^{1}$, Cole M. Haynes ${ }^{1}$, Jae-Hyuck Shim ${ }^{3 *}$

${ }^{1}$ Department of Molecular, Cell and Cancer Biology, University of Massachusetts Medical

School, Worcester, Massachusetts, USA

${ }^{2}$ Department of Biological Chemistry and Molecular Pharmacology, Harvard Medical School,

Boston, Massachusetts, USA

${ }^{3}$ Department of Medicine/Division of Rheumatology, University of Massachusetts Medical

School, Worcester, Massachusetts, USA

*Correspondence:

Xianpeng Ge

Email: paul.ge@umassmed.edu

or

Jae-Hyuck Shim

Email: jaehyuck.shim@umassmed.edu 


\begin{abstract}
The endocytic pathway actively interacts with mitochondria in maintaining cellular homeostasis. However, how the dysfunction of this inter-organelle interaction causing pathological outcomes remains less understood. Here we show that an aberrant endocytic pathway from the deficiency of CHMP5 in skeletal progenitor cells causes accumulation of functionally compromised mitochondria, which induce cellular senescence via reactive oxygen species (ROS)-mediated oxidative stress and DNA damage. These senescent progenitors can lead to distorted skeletal growth via a combination of cell-autonomous and non-autonomous mechanisms. Consequently, mice lacking Chmp5 in Ctsk-expressing periskeletal progenitors or Dmpl-expressing musculoskeletal progenitors develop multiple skeletal/muscular abnormalities, including robust bone overgrowth, progressive joint stiffness, and myopathy. Targeting senescent cells using senolytic drugs significantly alleviates these lesions and improves animal motility. Overall, our results reveal that CHMP5 restricts skeletal progenitor cell senescence through maintaining the endo-lysosomal-mitochondrial network and cell senescence represents a yet unexplored mechanism for detrimental alterations from the perturbed organelle network.
\end{abstract}


The endosomal sorting complex required for transport (ESCRT) machinery is an evolutionarily conserved molecular mechanism essential for diverse cell biological processes. Since it was initially discovered in the early 21 st century in the budding yeast Saccharomyces cerevisiae, the molecular gear has been predominantly dissected in the endocytic pathway, which sorts ubiquitinated membrane proteins and bound cargoes committing to lysosomal degradation ${ }^{1-4}$. In addition to the endocytic process, ESCRT proteins also play critical roles in other cellular activities, such as exocytosis, vial budding, cytokinesis, neuron pruning, plasma membrane repair, and nuclear envelope assembly ${ }^{2,4}$. In the endocytic pathway, the ESCRT machinery consists of a series of functionally overlapping protein complexes, including ESCRT-0, -I, -II, and -III. While ESCRT-0, -I, and -II complexes are mainly responsible for protein sorting and recruiting ESCRT-III components, the ESCRT-III complex, cooperating with the ATPase VPS4, is the primary executor of membrane severing during intraluminal vesicle formation of multivesicular bodies (MVBs) ${ }^{3,5}$.

The charged MVB protein CHMP5, the mammalian ortholog of yeast VPS60/MOS10, is a component of the ESCRT-III protein complex and plays an essential role in the late stage of MVB formation ${ }^{6}$. As yeast $\operatorname{Vps} 60 / \mathrm{Mos} 10$-null mutation disturbs the endosome-to-vacuole

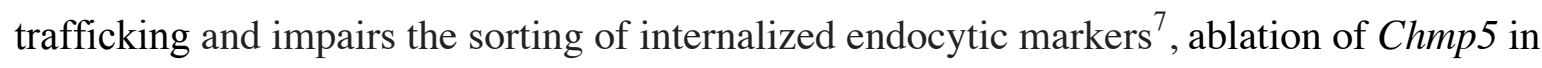
mouse embryonic fibroblasts (MEFs) also affects the sorting from late endosomes/MVBs to lysosomes and reduces lysosomal degradation of multiple activated receptors ${ }^{6}$. Notably, recent studies showed that Chmp5 deficiency in osteoclasts and T lymphocytes does not affect the endocytic pathway ${ }^{8,9}$. Since osteoclasts and T lymphocytes are of hematopoietic origin and 
MEFs belong to the mesenchymal cell lineage, we hypothesized that the regulation of the endocytic pathway by CHMP5 might be cell lineage-specific.

The endocytic pathway constantly communicates with mitochondria by transporting iron and lipids into these organelles ${ }^{10,11}$, regulating mitochondrial dynamics ${ }^{12,13}$, and monitoring mitochondrial quality control via taking in mitochondrial-derived vesicles or degrading entire damaged mitochondria ${ }^{14,15}$. Nevertheless, how a disturbed communication between the endocytic pathway and mitochondria causing pathological conditions remains less appreciated. In this study, by deleting Chmp5 using genetic mouse models and in vitro CRISPR/CAS9 technology, we demonstrate that Chmp5 deficiency causes accumulation of MVB- and lysosome-like compartments and impairs the function of lysosomal degradation in skeletal progenitor cells. Furthermore, the aberrant endocytic pathway leads to an increased abundance of dysfunctional mitochondria, which induces cellular senescence through ROS-mediated oxidative stress and DNA damage. These senescent progenitors can affect the proliferation and differentiation of neighboring wild-type progenitors via a paracrine mechanism, cooperatively resulting in multiple senescence-associated musculoskeletal abnormalities. Targeting senescent cells using senolytic drugs can mitigate these lesions. These results highlight that CHMP5 is indispensable for the endocytic pathway in skeletal progenitors and cell senescence underlies pathological changes from the perturbed endo-lysosomal-mitochondrial network. 


\section{Results}

\section{Ablation of Chmp5 in Ctsk-expressing skeletal progenitors causes aberrant bone growth}

Apart from the well-characterized roles in osteoclasts, the Ctsk gene has recently been reported to identify periskeletal and cranial suture progenitor cells ${ }^{16-18}$. Notably, the ablation of Chmp 5 in mouse Ctsk-expressing cells (hereafter referred to as $C h m p 5^{\text {Ctsk }}$ mice) caused dramatic periskeletal bone overgrowth and suture patency compared to Chmp5 $5^{\mathrm{Ctsk} /+}$ and $\mathrm{Chmp} 5^{\mathrm{fl} / \mathrm{ll}}$ littermate controls (Fig. 1a-c and Supplementary Fig. 1a), in addition to the previously reported increased osteoclast differentiation and activity ${ }^{9}$. The aberrant bone growth in Chmp5 $5^{\text {Ctsk }}$ mice became distinct around postnatal day $10(\mathrm{~d} 10)$, progressed with age, and involved multiple bones and joints across the body, including the knee, ankle, and foot joints, and the temporomandibular joint (Fig. 1a-c and Supplementary Fig. 1b). With age, these animals developed severe skeletal deformation, progressive joint stiffness, reduced motility, and dwarfism.

To exclude the possibility of osteoclasts as the source of periskeletal and suture lesions in Chmp $5^{\text {Ctsk }}$ mice, we performed tartrate-resistant acid phosphatase (TRAP) staining to detect osteoclasts at the early stage of these lesions (postnatal d10 to d14), which showed no TRAP ${ }^{+}$ osteoclasts at these locations (Fig. 1c and Supplementary Fig. 1c). Also, anti-resorptive treatments using Zolendronic acid or OPG-Fc could not prevent the periskeletal bone overgrowth and suture patency, although trabecular bone mass was substantially increased in both Chmp5 $5^{\text {Ctsk }}$ and $C h m p 5^{f l / l}$ mice (Supplementary Fig. 1d-h). These results indicate that osteoclasts are not the main source for periskeletal and suture lesions in $\mathrm{Chmp5} 5^{\mathrm{Ctsk}}$ mice. By contrast, lineage tracing of $C t s k^{+}$skeletal progenitors using the dual fluorescence reporter mouse line 
$C h m p 5^{C t s k} ; \operatorname{Ros} a 26^{m T m G /+}$, in which the plasma membrane of Ctsk-expressing progenitors and their descendants is green $\left(\mathrm{GFP}^{+}\right)$, displayed enriched $\mathrm{GFP}^{+}$cells in the periskeletal and suture abnormalities (Fig. 1d, e and Supplementary Fig. 1i), suggesting that $C t s k^{+}$skeletal progenitors directly contribute to the development of these lesions in $C h m p 5^{C t s k}$ mice.

Notably, the manifestations of periosteal bone overgrowths, progressive joint stiffness, and impaired closure of craniofacial sutures in $C h m p 5^{\text {Ctsk }}$ mice are similar to characteristics of the rare human disorder cranio-osteoarthropathy, which is associated with loss-of-function mutations of the 15-hydroxyprostaglandin dehydrogenase (HPGD) gene that result in elevated levels of prostaglandin E2 (PGE2) production ${ }^{19,20}$. Ctsk $^{+}$skeletal progenitors (CD45; $\mathrm{CD}^{-} 1^{-}$;GFP ${ }^{+}$) sorted

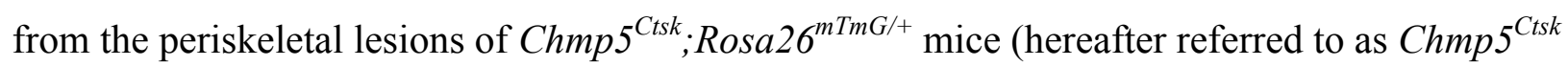
skeletal progenitors) demonstrated a significant decrease in the expression of Hpgd gene compared to those from $\mathrm{Chmp5}^{\mathrm{Ctsk/+}} ; \operatorname{Rosa}_{2} 6^{m T m G /+}$ control mice (Fig. 1f-h and Supplementary Fig. 1j). Also, Ptgs 2 and $A b c c 4$ genes, which are responsible for PGE2 production and export respectively, were upregulated in $C h m p 5^{C t s k}$ skeletal progenitors, while Ptgs 1 gene, a regulator of physiological PGE2 synthesis, showed no significant change (Fig. 1h). These results show that Chmp5 $5^{\text {Ctsk }}$ mice recapitulate phenotypic and genetic features of the human disorder cranioosteoarthropathy and suggest a role of CHMP5 in regulating the PGE2 pathway.

\section{Ablation of Chmp5 in Dmpl-expressing progenitors causes abnormal bone expansion and myopathy}

We next sought to verify the role of Chmp5 in skeletal progenitors by deleting this gene using another skeletal progenitor cell-specific Cre mouse line. As deletion of Chmp5 in Prrxl- 
expressing limb mesenchymal progenitors ${ }^{21}$ (Chmp5 $5^{\text {Prrxl }}$ mice), Col2-expressing perichondral progenitors $^{22}$ (Chmp $5^{\mathrm{Col} 2}$ mice), or $\mathrm{Osx}$-expressing osteoprogenitors ${ }^{23}$ (Chmp $5^{\mathrm{Osx}}$ mice) caused embryonic lethality, we employed the Dmp1-Cre mouse line which also labels postnatal endosteal osteoprogenitors in addition to mature osteoblasts and osteocytes ${ }^{24,25}$. The fatemapping data in Dmp1-Cre; Rosa $26^{m T m G /+}$ reporter mice affirmed the localization of Dmplexpressing cells on the surface of endosteum and trabecular bone from the early stage of postnatal growth (Supplementary Fig. 2a). Also, in an attempt to isolate colony-forming $\mathrm{Dmpl}^{+}$ $\left(\mathrm{CD} 45^{-} ; \mathrm{CD}^{-} 1^{-} \mathrm{GFP}^{+}\right)$cells from bones of Dmp1-Cre; Rosa $26^{m T m G /+}$ mice, we found that these cells were easy to get at 3 weeks of age, but not from neonatal mice (P0), providing further evidence that DMP1 labels postnatal osteoprogenitors.

Unlike $C h m p 5^{\text {Prrxl }}, C h m p 5^{\text {Col2 }}$, and $C h m p 5^{O s x}$ mice, $C h m p 5^{\text {Dmpl }}$ mice were born at normal Mendelian ratios and were viable. Within three weeks of age, skeletons in Chmp $5^{D m p l}$ mice started to display distinct expansion in comparison with $C h m p 5^{D m p 1 /+}$ and $C h m p 5^{f l f l}$ littermate controls, which progressed with age and became much severe at one year of age (Fig. 2a). The skeletal alterations in $C h m p 5^{\text {Dmpl }}$ mice were further analyzed in detail at around the age of ten weeks (Fig. 2b-e and Supplementary Fig. 2b, c). Notably, except for the expansion of cortical bones, Chmp5 $5^{D m p l}$ mice also displayed reduced trabecular bone mass (Fig. 2c and Supplementary Fig. 2b, c), likely due to the increased osteoclast formation (Supplementary Fig. 2d). Intriguingly, the pattern of bone changes in $C h m p 5^{D m p l}$ mice is similar to that in Chmp $5^{\text {Ctsk }}$ mice in terms of the cortical bone expansion and decreased trabecular bone mass, although the bone expansion in $C h m p 5^{D m p l}$ animals mainly happened at the endosteum in contrast to that periosteal bone overgrowth in $C h m p 5^{\text {Ctsk }}$ mice. 
As Dmpl-expressing progenitors also generate skeletal muscle cells ${ }^{25}, C h m p 5^{D m p 1}$ mice displayed a gradual decrease of skeletal muscle mass and functions with age (Fig. 2a, f, $\mathbf{g}$ ). These Chmp $5^{\text {Dmpl }}$ mice also showed apparent, recurrent seizures starting around 8-10 weeks of age, which might be secondary to skeletal muscle dysfunction. Combined, these results indicate that CHMP5 is critical in regulating the functions of multiple lineages of musculoskeletal progenitors.

\section{Chmp5 deficiency induces cellular senescence and increases the osteogenic capacity of}

\section{skeletal progenitors}

To understand the mechanism of skeletal overgrowth in Chmp5-deficient mice, we hypothesized that depletion of Chmp5 in skeletal progenitors increases cell proliferation and osteogenesis which eventually led to the aberrant bone growth. Unexpectedly, Chmp5 $5^{\text {Ctsk }}$ skeletal progenitors isolated from the early periskeletal lesions displayed a significant decrease in cell proliferation rate compared to $C h m p 5^{C t s k /+}$ control cells (Fig. 3a). A reduced cell number during culture was also detected in $\mathrm{Chmp}^{\text {Ctsk }}$ calvarial suture progenitors at two weeks of age (Supplementary Fig. 3a). Likewise, $D m p 1^{+}$osteoprogenitors isolated from $C h m p 5^{D m p l} ; \operatorname{Ros} a 26^{m T m G /+}$ mice formed fewer numbers and smaller sizes of colonies compared to those from $C h m p 5^{\text {Dmpl/+ }} ; \operatorname{Rosa} 26^{m T m G /+}$ control mice (Supplementary Fig. 3b), reflecting that Chmp5 deficiency restricts the proliferation of $\mathrm{Dmpl}^{+}$osteoprogenitors as well. Furthermore, the in-situ terminal deoxynucleotidyl transferase dUTP nick end labeling (TUNEL) assay demonstrated more apoptotic cells in craniofacial sutures of $C h m p 5^{\text {Ctsk }}$ mice in comparison with $C h m p 5^{\text {Ctsk/+ }}$ mice 
(Supplementary Fig. 3c). However, unlike Chmp5 ${ }^{C t s k}$ suture progenitors, only approximately 5\% of Chmp5 ${ }^{\text {Ctsk }}$ periskeletal progenitors underwent apoptosis (Supplementary Fig. 3d).

To gain more insights into the mechanism of CHMP5 regulating cell fate, we performed a transcriptome analysis in $C h m p 5^{C t s k}$ and $C h m p 5^{C t s k /+}$ skeletal progenitors. The gene set enrichment analysis (GSEA) showed significant enrichment of genes associated with cell senescence in $C h m p 5^{\text {Ctsk }}$ relative to $C h m p 5^{\text {Ctsk/+ }}$ skeletal progenitors (Fig. 3b, gene list seeing Supplementary Table 1). Indeed, $C h m p 5^{\text {Ctsk }}$ mice also displayed characteristic phenotypes of accelerated senescence, such as hair loss, bone sclerosis, joint stiffness, and decreased motility (Fig. 3c). To further characterize the role of CHMP5 in regulating cellular senescence, we utilized CRISPR/CAS9 technology to delete Chmp5 in a periskeletal progenitor cell line $\mathrm{ATDC}^{26}$ (Supplementary Fig. 3e). Similar to $C h m p 5^{\text {Ctsk }}$ skeletal progenitors, Chmp5-deficient ATDC5 cells also displayed a reduced rate of cell proliferation along with a mild increase of cell apoptosis (Fig. 3d and Supplementary Fig. 3f, g). However, inhibition of cell apoptosis using the pan-caspase inhibitor Q-VD-Oph could not block the reduction in cell number (Supplementary Fig. 3h), suggesting that cell apoptosis is not the main cause for the reduced cell proliferation rate in Chmp5-deficient cells. Instead, the cell cycle analysis showed that lack of Chmp5 caused more cells accumulated at G0/G1 or G2/M phases and fewer cells at the S phase (Fig. 3e), indicating that Chmp5 deficiency induces cell cycle arrest, a characteristic feature of cell senescence. Together, these results demonstrate a role of CHMP5 in restricting cellular senescence in skeletal progenitors. 
Histologic analyses revealed that the bone overgrowth in $C h m p 5^{\text {Ctsk }}$ and $C h m p 5^{\text {Dmpl }}$ mice developed mainly through the intramembranous ossification as chondrocytes were rarely detected in these lesions (Fig. 1a, 2d and Supplementary Fig. 1c). Also, Chmp $5^{\text {Ctsk }}$ skeletal progenitors showed a molecular pattern of decreased chondrogenic potential (Supplementary

Fig. 3i, j), while the extracellular matrix mineralization was markedly increased when these cells were induced toward osteogenesis (Fig. 3f). Consistently, the ingenuity pathway analysis of transcriptome data showed increased activity of osteoblast differentiation-related pathways in Chmp5 ${ }^{\text {Ctsk }}$ relative to $C h m p 5^{\text {Ctsk/+ }}$ skeletal progenitors (Fig. 3g, gene list referring to Supplementary Table 2). Likewise, the deletion of Chmp5 in the calvarial osteoblast progenitor cell line MC3T3-E1 also increased the mineralization activity and decreased cell proliferation (Fig. 3h and Supplementary Fig. 3k). Combined, these results indicate that the cortical bone expansion in $C h m p 5^{C t s k}$ and $C h m p 5^{D m p l}$ mice partly results from the enhanced capacity of intramembranous ossification of Chmp5-deficient skeletal progenitors.

\section{Paracrine actions of Chmp5-deficient skeletal progenitors}

An apparent feature of skeletal lesions in $C h m p 5^{C t s k} ; \operatorname{Rosa} 26^{m T m G /+}$ mice is the integration of many cells lacking evidence of Cre-mediated recombination (GFP') into the periskeletal overgrowth (Fig. 1d), suggesting that Chmp5-deficient skeletal progenitors might recruit neighboring wild-type cells cooperatively facilitating the aberrant bone growth. Similarly, Chmp $5^{\text {Dmpl }}$ bone marrow stromal cells displayed an increased capacity of osteoclast differentiation (Supplementary Fig. 2d), indicating that $C h m p 5^{D m p 1}$ osteoprogenitors could affect nearby osteoclast formation via a paracrine mechanism. The GSEA of transcriptome data showed significant enrichment of genes associated with the senescence-associated secretory 
phenotype (SASP) in Chmp5-deficient cells (Fig. 4a, gene list seeing Supplementary Table 1). Notably, confocal fluorescence microscopy captured many extracellular vesicles shed from the plasma membrane of Chmp $5^{\text {Ctsk }}$ skeletal progenitors, which were rarely detected around wildtype $\mathrm{Ctsk}^{+}$skeletal progenitors (Fig. 4b). Furthermore, the nanoparticle tracking analysis demonstrated increased concentrations of extracellular vesicles in the culture medium of both Chmp $5^{\text {Ctsk }}$ skeletal progenitors and Chmp5-deficient ATDC5 cells (Fig. 4c-e and Supplementary Fig. 4a-c). These results show that Chmp5 deficiency in skeletal progenitors induces a phenotype of elevated secretion, a characteristic feature of senescent cells.

Moreover, ATDC5 cells treated with the conditioned medium collected from Chmp5-deficient cells showed increased proliferation compared to those treated with medium from Chmp5sufficient cells (Fig. 4f and Supplementary Fig. 4d). Likewise, wild-type skeletal progenitors $\left(\mathrm{CD} 45^{-} ; \mathrm{CD}^{-} 1^{-} \mathrm{GFP}^{-}\right.$in Fig. 1f) isolated from $\mathrm{Chmp5}^{\mathrm{Ctsk}} ; \mathrm{Rosa} 26^{m T m G /+}$ mice also showed increased proliferation along with enhanced osteogenic capacity in comparison with corresponding cells from Ctsk-Cre;Rosa $26^{m T m G /+}$ control mice (Fig. $\left.\mathbf{4 g}, \mathbf{h}\right)$. Immunostaining for Ki-67 demonstrated an increased number of proliferating cells in the periskeletal lesion of Chmp $5^{\text {Ctsk }}$ mice (Fig. 4i and Supplementary Fig. 4e). Lastly, genes expressing secreted factors that promote osteogenesis, including Tgf1 $\beta$, Wnt10b, Il6, Il11, Cthrc1, and Sema3a, was significantly upregulated in $C h m p 5^{\text {Ctsk }}$ skeletal progenitors (Supplementary Fig. 4f). These secreted factors, together with the elevated production of PGE2 (Fig. 1h), are likely responsible for the paracrine effects of Chmp5-deficient skeletal progenitors.

\section{Chmp5 deficiency perturbs the endocytic pathway in skeletal progenitors}


Next, we wondered whether the deficiency of Chmp5 in skeletal progenitors affects the endocytic pathway. Notably, approximately $45 \%$ of $C h m p 5^{\text {Ctsk }}$ skeletal progenitors contained enlarged $\mathrm{GFP}^{+}$vesicles which were rarely found in wild-type Ctsk ${ }^{+}$skeletal progenitors (Fig. 5a), suggesting a possible issue of the endocytic pathway in the Chmp5-deficient cells. Next, we utilized molecular markers for different components and the function of the endocytic pathway to dissect the influence of Chmp5 deficiency on the endo-lysosomal system (Fig. 5b). The tracing of LysoTracker Red DND-99 and immunostaining of lysosome-associated membrane protein 1 (LAMP1) showed positive stain in many of the enlarged vesicles in both $C h m p 5^{\text {Ctsk }}$ skeletal progenitors and Chmp5-deficient ATDC5 cells (Fig. 5c, d and Supplementary Fig. 5ac). The number and size of vesicles stained for the late endosome marker RAB7 were also markedly increased in the Chmp5-deficient cells relative to wild-type cells (Fig. 5e, f and Supplementary Fig. 5d). Meanwhile, many of these accumulated vesicles were double-positive for LAMP1 and RAB7 (Fig. 5g), suggesting that they are terminal compartments in the endocytic pathway. Accordingly, the transmission electron microscopy (TEM) demonstrated significant accumulation of MVB-like and lysosome-like structures with different electronic density in Chmp5 $5^{\text {Ctsk }}$ skeletal progenitors (Fig. 5h and Supplementary Fig. 5e).

To examine the function of the endocytic pathway, we used a $\mathrm{pH}$-sensitive fluorescent EGFconjugate and traced the degradation of internalized EGF receptor. The deficiency of Chmp5 significantly delayed the degradation of the EGF conjugate in Chmp $5^{\text {Ctsk }}$ skeletal progenitors (Fig. 5i), indicating an impaired function of the endocytic pathway. Of note, the EEA $1^{+}$earlyendosomes, $\mathrm{RAB} 11^{+}$recycling endosomes, and TGN $38^{+}$trans-Golgi network were relatively normal in the Chmp5-deficient cells (Supplementary Fig. 5f-h). Together, these results show 
that CHMP5 is indispensable for the endocytic pathway in skeletal progenitors. Lack of this gene causes the accumulation of late endosomes/MVBs and lysosomes and reduces the activity of lysosomal degradation.

\section{Chmp5 deficiency causes mitochondrial dysfunction to induce cell senescence}

How could the aberrant endocytic pathway cause cellular senescence? The endocytic pathway, especially late endosomes/MVBs and lysosomes, is critical in mitochondrial quality control through degrading toxic mitochondrial proteins or entire damaged mitochondria ${ }^{14,15}$. Therefore, it is possible that the abnormal endocytic pathway after Chmp5 depletion compromises these mitochondrial quality surveillance routes and leads to the dysfunction of mitochondria, which are important regulators of cellular senescence ${ }^{27,28}$. The GSEA of transcriptome data showed significant enrichment of genes associated with the oxidative stress-induced cell senescence in Chmp $5^{\text {Ctsk }}$ skeletal progenitors (Fig. 6a; gene list referring to Supplementary Table 1). Accordingly, increased levels of mitochondrial ROS, which is the main mediator of cellular oxidative stress that induces senescence ${ }^{28}$, were detected in these Chmp5-deficient cells (Fig. 6be). Chmp5 deficiency also increased the abundance of mitochondria (Fig. 6f, g and Supplementary Fig. 6a), although mitochondrial functions were compromised as shown by reduced tolerance to galactose, decreased mitochondrial spare respiratory capacity, and increased extracellular acidification rate in Chmp5-deficient cells (Fig. 6h, i). The TEM showed that mitochondrial morphology was comparable between $C h m p 5^{\text {Ctsk }}$ and wild-type skeletal progenitors (Fig. 6j and Supplementary Fig. 6b).

Mitochondrial dysfunction and DNA damage response usually form a feedback loop to drive phenotypes of senescent cells ${ }^{28-30}$. The GSEA of transcriptome data also showed enrichment of 
genes associated with DNA damage-induced cell senescence in Chmp5-deficient cells (Fig. 6k, gene list seeing Supplementary Table 1). The upregulation of two critical DNA damageresponsive genes $H 2 a f x$ and $\operatorname{Trp} 53$ were confirmed by gene expression analysis in Chmp $5^{\text {Ctsk }}$ versus Chmp $5^{\text {Ctsk/+ }}$ skeletal progenitors (Fig. 61). Taken together, these results show that the aberrant endocytic pathway after Chmp5 deletion downgrades the mitochondrial quality control system and subsequently causes accumulation of functionally compromised mitochondria, which induce skeletal progenitor cell senescence via ROS-mediated oxidative stress and DNA damage.

\section{Targeting senescent cells mitigates the bone overgrowth and myopathy}

Next, we evaluated if releasing the cellular oxidative stress in senescent skeletal progenitors could alleviate musculoskeletal lesions in $C h m p 5^{C t s k}$ and $C h m p 5^{D m p 1}$ mice. Firstly, in vitro treatment with the antioxidant $N$-Acetylcysteine (NAC) reversed the reduced activity of cell proliferation, but could not alter the increased mineralization during osteogenic induction, in Chmp5-deficient ATDC5 cells and Chmp5 ${ }^{\text {Ctsk }}$ skeletal progenitors respectively (Fig. 7a, b). Notably, NAC markedly inhibited the mineralization activity in wild-type Ctsk $^{+}$skeletal progenitors (Fig. 7b). However, the in vivo treatment of $C h m p 5^{C t s k}$ mice with NAC unanticipatedly exacerbated skeletal lesions and resulted in premature death around the weaning age, while littermate control mice treated with the same dose of NAC did not demonstrate any gross phenotypes.

To test whether eliminating senescent cells could mitigate the musculoskeletal lesions, animals were treated using senolytic drugs quercetin and dasatinib $(\mathrm{Q}+\mathrm{D})$ starting from the first two weeks after birth. Impressively, the treatment with senolytic drugs Q + D weekly for 7 weeks 
significantly decreased the periskeletal bone overgrowth and improved animal motility in $C h m p 5^{C t s k}$ mice, although the body size remained smaller compared to $C h m p 5^{f l f l}$ control mice (Fig. 7c-f and Supplementary Fig. 7, 8a). Similarly, treating Chmp $5^{D m p l}$ mice with Q + D also improved musculoskeletal symptoms and partly restored skeletal muscle functions (Fig. 7g and Supplementary Fig. 8b). Notably, the recurrent seizures in these animals disappeared. There were no apparent abnormalities in $C h m p 5^{f l f l}$ control animals treated with the same dose of Q + D in parallel. Together, these results demonstrate that eliminating senescent cells using senolytic drugs is effective to mitigate the bone overgrowth and myopathy in $C h m p 5^{\text {Ctsk }}$ and $C h m p 5^{\text {Dmp I }}$ mice. 


\section{Discussion}

The intracellular organelles, like endosomes, lysosomes, and mitochondria, form an elaborate functional network in maintaining cellular homeostasis. The ESCRT machinery-mediated endocytic pathway is tightly connected with mitochondria in physiological and pathologic circumstances. Our results show that the ESCRT protein CHMP5 is critical in maintaining the integrity of the endo-lysosomal-mitochondrial network in skeletal progenitors. CHMP5 deficiency disturbs the endocytic pathway and causes accumulation of dysfunctional mitochondria, which induce cellular senescence and result in severe pathological outcomes via a combination of cell-autonomous and non-autonomous mechanisms. Eliminating senescent cells using senolytic drugs is effective to mitigate these lesions. These results suggest that accelerating stem/progenitor cell senescence represents an underlying mechanism for disorders caused by the aberrant endo-lysosomal-mitochondrial network.

In both skeletal progenitors and MEFs, which are non-hematopoietic cells, CHMP5 is indispensable for the endocytic pathway, supporting a cell lineage-specific role of CHMP5 in regulating the endocytic pathway. Therefore, our finding that CHMP5 preserves the endolysosomal-mitochondrial network could be beyond skeletal progenitors and also happen in other cell types in the musculoskeletal system or cells from other non-hematopoietic tissues/organs. Since CHMP5 regulates the activation of ATPase VPS4 which catalyzes the disassembly of ESCRT-III complex from late endosomes/MVBs ${ }^{31,32}$, loss of CHMP5 might impair the membrane remodeling of late endosomes and block their fusion with lysosomes. In fact, Chmp5 is necessary for efficient fusion of late endosomes and lysosomes in MEFs ${ }^{6}$. Notably, the characteristics of TEM in Chmp5-deficient skeletal progenitors are similar to those of cells from 
human inclusion-cell disease, a lysosomal storage disease with defects in almost all lysosomal enzymes and manifesting bone, joint, and muscle problems ${ }^{33,34}$. As the endocytic pathway also participates in sorting and processing hydrolases during lysosomal biogenesis ${ }^{35}$, the perturbation of the endocytic pathway may affect lysosomal enzyme maturation and activation. Indeed, the Vps60/Mos 10 mutation in yeast impairs the processing of the vacuolar protease $\mathrm{CPY}^{7}$, which is homologous to human lysosomal serine carboxypeptidase Cathepsin A. Thus, the Chmp5deficient mice also recapitulate partial manifestations of the lysosomal storage disease.

Except for that the endocytic pathway mediates mitochondrial quality $\operatorname{control}^{14,15,36}$, dysfunction of endosomes, lysosomes, and mitochondria has been simultaneously observed in multiple human diseases especially in lysosomal storage diseases ${ }^{37,38}$. Our results in this study establish a direct connection between the aberrant endocytic pathway and mitochondrial dysfunction and reveal that cell senescence underlies the resulting musculoskeletal pathologies. Additionally, the increased abundance and impaired functions of mitochondria have been established in multiple in vitro and in vivo models of cell senescence ${ }^{28-30}$, in which the accumulation of dysfunctional mitochondria produce elevated levels of ROS, causing persistent cellular oxidative stress and DNA damage and forming a feedback loop to accelerate cell senescence. These, together with our findings in this study, support that the mitochondrion is a major player in regulating cell senescence.

Resembling the paracrine effect of Chmp5-deficient cells, inactivation of other ESCRT proteins, such as VPS25 and TSG101, also causes non-autonomous cell survival and overgrowth ${ }^{39-41}$. In Drosophila, Vps25 mutations cause cell-autonomous death and non-autonomous cell 
proliferation. Blocking the cell death induces massive overgrowth of vps 25 mosaics $^{39}$. This is similar to our results in $C h m p 5^{\text {Ctsk }}$ mice that pro-survival treatment with NAC exacerbates skeletal lesions and causes premature death, which suggests that the secretory phenotype plays a prominent role in the development of pathological alterations. Inhibition of the secretory phenotype by eliminating the "zombie" cells using senolytic drugs significantly alleviate musculoskeletal lesions in $C h m p 5^{C t s k}$ and $C h m p 5^{D m p l}$ mice. In terms of the secretory phenotype of Chmp5-deficient cells, it is noteworthy that accumulated MVBs, lysosomes, and mitochondria, as well as their components, can be directly secreted out of the cell and contribute to the secretome ${ }^{42-44}$, which is probably beyond a typical SASP.

There are some limitations to our present study. First of all, although our results elucidate a definite role of CHMP5 in skeletal progenitors, we can't exclude that Chmp5 deficiency in progenies of $\mathrm{Ctsk}^{+}$and $\mathrm{Dmpl}^{+}$progenitors also contributes to musculoskeletal phenotypes in Chmp $5^{\text {Ctsk }}$ and $C h m p 5^{\text {Dmpl }}$ mice respectively. In addition, osteoclasts in $C h m p 5^{\text {Ctsk }}$ mice can also cause aberrant bone growth ${ }^{9}$. Secondly, given the imperfect recombination efficiency and intrinsic nature of the mouse Cre-loxP system, the Chmp5-deficient and wild-type skeletal progenitors we isolated in this study could be heterogeneous cell populations. To conquer this potential pitfall, we have validated the results by deleting Chmp5 in two skeletal progenitor cell lines ATDC5 and MC3T3-E1. Also, it is not clear at present whether cell senescence is related to the gene dosage of Chmp5 loss. Thirdly, our results show that multiple bone coupling factors and the PGE2 production are upregulated in Chmp5-deficient skeletal progenitors. These secreted factors could be responsible for the paracrine effects of Chmp5-deficient cells. However, a secretome analysis is warranted to comprehensively understand the secretory phenotype in the 
following study. Future studies are also necessary to clarify the molecular signaling in neighboring wild-type cells that mediates their proliferation and osteogenesis.

In conclusion, the ESCRT protein CHMP5 restricts cellular senescence by preserving the endolysosomal-mitochondrial network in skeletal progenitors. Deficiency in CHMP5 or its regulated organelle network can change cell fate and cause detrimental changes to tissues and organisms. Since Chmp5-deficient mice recapitulate features of human disorders cranio-osteoarthropathy and lysosomal storage diseases, it is rational to start evaluating the role of cell senescence in, and therapeutic potential of targeting senescent cells for, these intractable clinical conditions. 


\section{Acknowledgments}

We thank Dr. S. Ghosh for providing Chmp5-floxed mice; Ms. Y. Yang for assistance in acquiring micro-CT data; Ms. H. Zou for genotyping mice; the Flow Cytometry Core Lab, Core Electron Microscopy Facility, and Confocal Core at UMass Medical School for technical help; Drs. Gregory Hendricks and Lara Strittmatter for help in interpreting TEM data; Drs. T. Shpilka for assistance in running the Seahorse analyzer; Drs. G. Yu and J. Park for participating in the transcriptome data analysis. The project was partly supported by NIH grants (S10RR027897, S10OD021580). J.H.S holds support from NIAMS of the NIH (R01AR068983, R21AR073331) and the AAVAA Therapeutics.

\section{Contributions}

X.G. perceived the project, designed and executed all experiments, analyzed and interpreted the results, and wrote the manuscript. L.H. produced the CRISPR/CAS9 lentivirus. H.L. contributed to part of transcriptome data analyses. C.M.H. offered critical suggestions and main reagents for mitochondrial analyses and supported X.G. to finish the study. J.H.S. financially supported the study and involved in the manuscript preparation.

\section{Competing interests}

J.H.S. is a scientific co-founder of AAVAA Therapeutics and holds equity in this company. Other authors declare no competing interests. 


\section{Methods}

\section{Reagents and Cell Lines}

Rabbit anti-CHMP5 polyclonal antibody ${ }^{6}$

Mouse anti-EEA1 antibody (clone 14/EEA1, BD Bioscience)

Rabbit anti-RAB7 antibody (clone D95F2, Cell Signaling Technology)

Rabbit anti-RAB11 antibody (clone D4F5, Cell Signaling Technology)

Rat anti-CD107a (LAMP1) antibody (clone 1D4B, BD Bioscience)

Rat anti-TGN38 antibody (clone 831629, R\&D Systems)

Mouse anti-OXPHOS antibody cocktail (cat\#ab1 10413, Abcam)

Rabbit anti-Ki67 antibody (cat\# ab15580, Abcam)

Mouse anti-GAPDH antibody (clone GT239, GeneTex)

Mouse anti-beta-actin antibody (clone 2F1-1, Biolegend)

APC anti-mouse CD45 (clone 30-F11, Biolegend)

APC anti-mouse CD31 (clone 390, Biolegend)

Goat anti-Rat secondary antibody, Alexa Fluor 633 (cat\# A-21094, ThermoFisher)

Goat anti-Mouse secondary antibody, Alexa Fluor 633 (cat\# A-21050, ThermoFisher)

Goat anti-Rat secondary antibody, Alexa Fluor 488 (cat\# A-11006, ThermoFisher)

Goat anti-Mouse secondary antibody, Alexa Fluor 488 (cat\# A-11001, ThermoFisher)

Goat anti-Rat secondary antibody, Alexa Fluor 594 (cat\# A-11007, ThermoFisher)

Goat anti-Mouse secondary antibody, Alexa Fluor 594 (cat\# A-11005, ThermoFisher)

Goat anti-Rabbit secondary antibody, Alexa Fluor ${ }^{\circledR} 594$ (cat\# A-1 1012, ThermoFisher)

In Situ Apoptosis Detection Kit (cat\#ab206386, Abcam)

LysotrackerTM Red DND-99 (cat\# L7528, ThermoFisher) 
pHrodo $^{\text {TM }}$ Red Epidermal Growth Factor (EGF) Conjugate (cat\# P35374, ThermoFisher)

Tetramethylrhodamine, Ethyl Ester, Perchlorate (TMRE, cat\# T669, ThermoFisher)

MitoTrackerTM Green FM (cat\# M7514, ThermoFisher)

CellROX ${ }^{\mathrm{TM}}$ Deep Red Reagent (cat\# C10422, ThermoFisher)

N-Acetyl-L-cysteine (cat\# A7250, Sigma-Aldrich)

Quercetin (cat\# Q4951, Sigma-Aldrich)

Dasatinib (cat\# CDS023389, Sigma-Aldrich)

Polyethylene glycol 400 (PEG400, Fisher Scientific)

Annexin V Apoptosis Detection Kit APC (cat\# 88-8007-72, ThermoFisher)

APC BrdU Flow Kit (cat\# 552598, BD Bioscience)

InSolution $^{\mathrm{TM}}$ Q-VD-OPh, Non-O-methylated (cat\# 551476, Millipore)

VECTASTAIN® Elite ${ }^{\circledR}$ ABC HRP Kit (Peroxidase, Rabbit IgG; cat\# PK-6101, VECTOR

Laboratories)

Recombinant Mouse TRANCE/RANK L/TNFSF11 Protein (cat\# 462-TR, R\&D Systems)

Recombinant Mouse M-CSF Protein (cat\# 461-ML, R\&D Systems)

alamarBlue ${ }^{\mathrm{TM}}$ Cell Viability Reagent (cat\# DAL1025, ThermoFisher)

Seahorse XF Cell Mito Stress Test Kit (cat\# 103015-100, Agilent)

ALIZARIN red 2\% solution pH 4.2 (cat\# 50-317-34, Fisher scientific)

Zolendronic acid monohydrate (cat\# SML0223, Sigma-Aldrich)

OPG-Fc (Amgen Inc.)

MC3T3-E1 cells (mouse C57BL/6 calvarial fibroblasts; cat\# 99072810, Sigma-Aldrich)

ATDC5 cells (mouse 129 teratocarcinoma-derived osteochondral progenitors; cat\# 99072806, Sigma-Aldrich) 


\section{Mice}

The Chmp5 $5^{f l o x}$, Ctsk-Cre, Dmpl-Cre, Prrx1-Cre, Col2-Cre, and Osx-Cre mice are previously reported $^{9,45-47}$. The Rosa $26^{m T m G}$ mouse was purchased from Jackson Laboratories. To generate experimental animals, Chmp $5^{f l f l}$ mice were crossed with $C h m p 5^{C t k s /+}$ and $C h m p 5^{\text {Dmpl/t }}$ mice respectively. For generating reporter mouse lines, $C h m p 5^{C t k s /+}$ and $C h m p 5^{D m p l /+}$ mice were mated with $C h m p 5^{f l f f l} ; \operatorname{Rosa} 26^{m T m G}$ mice; Ctsk-Cre and Dmp1-Cre mice were crossed with Rosa26 $6^{m T m G}$ animals respectively. All mice were kept C57BL/6J background and housed in the standard animal facility on a 12-hour light/dark cycle with ad libitum access to water and normal chow. In most situations, littermates were compared otherwise as indicated. All animals were used in accordance with the NIH Guide for the Care and Use of Laboratory Animals and were handled according to protocols approved by the University of Massachusetts Medical School on animal care (IACUC).

\section{Skeletal Progenitor Cell Isolation and Sorting}

The periskeletal progenitors were isolated from hindlimbs of Ctsk-Cre;Rosa2 $6^{m T m G /+}$, $C h m p 5^{C t s k /+} ; \operatorname{Rosa} 26^{m T m G /+}$, and $C h m p 5^{C t s k} ; \operatorname{Rosa}_{26^{m T m G /+}}$ mice at 2 weeks of age according to the previously established method ${ }^{26}$. Briefly, the periskeletal tissues were dissected, minced into small pieces, and digested with $1 \mathrm{mg} / \mathrm{ml}$ collagenase type I (Worthington), $1 \mathrm{mg} / \mathrm{ml}$ collagenase type II (Worthington) and $1.5 \mathrm{mg} / \mathrm{ml}$ dispase (Roche) for $30 \mathrm{~min}$ at $37^{\circ} \mathrm{C}$. Suture progenitors were acquired from the suture mesenchyme of the same animals, digested using 1 $\mathrm{mg} / \mathrm{ml}$ collagenase types I and II (1:3), and collected every $15 \mathrm{~min}$. Dmp $1+$ osteoprogenitors were detached from the whole bone of $C h m p 5^{\text {Dmpl }} ; \operatorname{Rosa} 26^{m T m G /+}$ and 
Chmp $5^{\text {Dmpl/+ }} ; \operatorname{Ros} a 26^{m T m G /+}$ mice at P0 and 3 weeks of ages using the same digestion protocol as periskeletal progenitors. Cells were cultured for one week, stained with anti-CD31 and anti-CD45 antibodies, and sorted using a FACSAria II cell sorter (BD Biosciences).

\section{Micro-CT and Radiography}

Micro-CT analyses were performed using the Scanco $\mu \mathrm{CT}-35, \mu \mathrm{CT}-40$ (Scanco Medical) or Inveon CT scanner (Siemens). For comparing cortical and trabecular bone parameters in $C h m p 5^{\text {Dmpl }}$ and control mice, distal femurs were scanned. Morphometric analysis for trabecular bone began at the growth plate and extended proximally for 300 slices. Cortical bone thickness was determined at the femoral midshaft. Thresholds of 280 and 320 were used for trabecular and cortical bone analyses, respectively. X-ray images were acquired using X-Ray MX-20 Specimen (Faxitron) following the default program.

\section{Histology, Immunohistochemistry, and Immunofluorescence}

All samples were fixed in 10\% neutral formalin or 4\% PFA overnight for premature samples or 2 days for mature skeletal samples. Samples were decalcified with 14\% EDTA and serial sections were cut for all samples. At least five slides at certain interval (80-100 $\mu \mathrm{m})$ from each sample were selected for histological evaluation. Fate-mapping images were acquired using a Leica TCS SP5 II laser scanning confocal microscope.

Immunohistochemistry for Ki-67 was performed using the VECTASTAIN ${ }$ Elite ${ }^{\circledR}$ ABC HRP Kit (Peroxidase, Rabbit IgG; Vector Laboratories). Cells for immunofluorescence were cultured in $35 \mathrm{~mm}$ MatTek glass bottom dishes (No. 1.5 coverslip, MatTek Corporation). 
Briefly, cells were fixed in 4\% PFA for 15 min, blocked in 1\% BSA for 30 min, and incubated with primary antibodies for 2 hrs and subsequently fluorescence conjugated secondary antibodies for $1 \mathrm{hr}$. Images were acquired using the Leica TCS SP5 II laser scanning confocal microscope. Quantification of intracellular vesicles was performed using FIJI software (NIH).

\section{CRISPR/CAS9 Lentiviral Infection}

Pairs of CRISPR guide RNA oligos (mouse Chmp5 single guide RNAs [sgRNAs] targeting GGCTCCGCCACCTAGCTTGA and GTTTCGCTTTTCCGAAGAAT on exon 1 respectively) were annealed and cloned into the BsmBI sites of lentiCRISPR V2-puro vector (plasmid 52961, Addgene). CRISPR lentiviral plasmids and lentiviral packaging plasmids (pMDLg/pRRE, pRSV-Rev, and pMD2.G; Addgene) were transfected into HEK 293T cells. Supernatants were harvested and filtered through a $0.45-\mu \mathrm{m}$ filter 2.5 days after transfection. MC3T3-E1 and ATDC5 cells were infected with CRISPR lentivirus and selected with puromycin $(4.5 \mu \mathrm{g} / \mathrm{ml}$, Clontech) for 7 days. The depletion of Chmp5 was confirmed by western blotting.

\section{Cell Cycle, Proliferation, and Apoptosis Analyses.}

Cell number was counted with a Countess II FL Automated Cell Counter (ThermoFisher) at indicated time intervals. AlamarBlue cell viability assay was performed as previously

described ${ }^{26}$. Cell cycle was determined using the APC-BrdU Flow Kit (BD Biosciences). In vitro apoptosis analysis was carried out with the Annexin V-PI stain and in situ cell apoptosis was determined with TUNEL staining (Abcam).

\section{RNA-sequencing, Data Analysis, and Functional Annotation}


Sorted Chmp5 $5^{\text {Ctsk }}$ and $C h m p 5^{\text {Ctsk/+ }}$ skeletal progenitors were subjected to RNA-sequencing (RNA-seq). For library preparation, $100 \mathrm{ng}$ of total RNA from each sample was subjected to rRNA depletion with NEBNext rRNA Depletion Kit (New England Biolabs) according to the manufacturer's manual. Subsequently, the rRNA-depleted RNA was used to build the RNA-seq library with NEBNext Ultra II Directional RNA Library Prep Kit (New England Biolabs) according to the manufacturer's manual. The index of each RNA-seq library was introduced by using NEBNext Multiplex Oligos for Illumina (New England Biolabs). Each RNA-seq library was analyzed by the fragment analyzer (Advanced Analytical Technologies) and quantified by KAPA Library Quantification Kit (KAPA Biosystems) according to the manufacturer's manual. Equal amount of each RNA-seq library was mixed for sequencing in one lane of Illumnia HiSeq3000 in paired-end 150 base mode (Illumina).

Raw Illumina pair-end reads were first aligned to ribosomal RNA (GenBank ID BK000964.1) with Bowtie2 v2.2.6 ${ }^{48}$. Reads that failed to map to ribosomal RNA were aligned to the mouse reference genome mm10 with STAR v2.5.3 ${ }^{49}$. Differential analysis was performed with Cuffdiff v2.2.1 ${ }^{50}$. The raw data were analyzed by two independent statisticians and similar results were obtained.

Pathway analyses were done for differentially expressed genes using the Reactome online analysis tool ${ }^{51}$ and the Ingenuity Pathway Analysis software (Qiagen). Gene ontology term overrepresentation analysis was run using PANTHER (version 14.1) with all genes expressed in a tissue or cell type as background and the differentially expressed gene list as the input. P-values from Fisher's exact tests were corrected by controlling the false discovery rate (FDR). Gene set 
enrichment analyses were performed using GSEA or ConsensusPathDB. For GSEA-based analysis, gene sets of interests were downloaded from the MsigDB, while all built-in gene sets were used for ConsensusPathDB-based analysis.

\section{In Vitro Osteogenesis and Osteoclastogenesis}

For osteogenic differentiation, $1.0 \times 10^{5}$ cells were plated into each well of 24 -well plates and cultured overnight. Cells were change into osteogenic media the next day ( $\alpha \mathrm{MEM}$ supplemented with 10\% FBS, $10 \mathrm{nM}$ dexamethasone, $50 \mu \mathrm{g} / \mathrm{ml} \mathrm{L}$-ascorbic acid, and 10mM $\beta$ glycerophosphate) and induced for indicated times. The osteogenesis was determined by alizarin red and von Kossa staining and examining the activity of alkaline phosphatase.

Osteoclastogenesis was performed using plastic-adherent cells from bone marrow of both adult (8-10 weeks of age) and young (3 weeks of age) $C h m p 5^{D m p 1 /+}$ and $C h m p 5^{D m p 1}$ mice. Bone marrow cells were plated in four replicates in 24-well plates at a density of $5 \times$ $10^{4}$ cells/well with $10 \mathrm{ng} / \mathrm{ml}$ of M-CSF (R\&D Systems). Three days later, cells were cultured in osteoclast medium supplemented with $10 \mathrm{ng} / \mathrm{ml}$ of M-CSF and $5 \mathrm{ng} / \mathrm{ml}$ of murine RANKL

(R\&D Systems) for 7-10 days. Fresh medium was changed every three days. Osteoclasts were quantified by counting TRAP-positive cells with 3 or more nuclei. Images were taken using a EVOS XL cell imaging system (ThermoFisher).

\section{Quantitative PCR and Western Blotting}

Primer sequences were used for qPCR are as following:

\section{Chmp5}

forward, 5'-ATGAGAGAGGGTCCTGCTAAG-3', 
reverse, 5'-CCGTGGTCTTGGTGTCCTTTA-3';

Hpgd

forward, 5'-GTGAACGGCAAAGTGGCTCT-3', reverse, 5'-TCCAATCCACCAATGCTACCT-3';

Ptgs 1

forward, 5'-TTACTATCCGTGCCAGAACCA-3', reverse, 5'-CCCGTGCGAGTACAATCACA-3';

Ptgs 2

forward, 5'-TGAGCAACTATTCCAAACCAGC-3', reverse, 5'-GCACGTAGTCTTCGATCACTATC-3';

Abcc4

forward, 5' - AGGAGCTTCAACGGTACTGG-3', reverse, 5'- GCCTTTGTTAAGGAGGGCTTC-3';

H2afx

forward, 5'-GTGGTCTCTCAGCGTTGTTC-3', reverse, 5'-CGGCCTACAGGGAACTGAA-3';

Tp53

forward, 5'-GTCACAGCACATGACGGAGG-3', reverse, 5'-TCTTCCAGATGCTCGGGATAC-3';

Gapdh

forward 5'-TGCCAGCCTCGTCCCGTAGAC-3', reverse 5'-CCTCACCCCATTTGATGTTAG-3'.

Primer sequences for $\operatorname{Col} 2 a 1, \operatorname{Sox} 9$, and Hprt are as previously reported ${ }^{26}$.

Total RNA was isolated from cells using the Trizol reagent (Qiagen). RNA samples were treated with the TURBO DNA-free ${ }^{\mathrm{TM}}$ Kit (Thermo Fisher) and equal amounts $(1-2 \mu \mathrm{g})$ were used for reverse transcriptase reaction using an iScript ${ }^{\mathrm{TM}}$ Reverse Transcription Supermix (Bio-Rad). qPCR was run using the SYBR ${ }^{\circledR}$ Green Master Mix on a CFX Connect ${ }^{\mathrm{TM}}$ RealTime PCR Detection System (Bio-Rad). Gene expression was relative to the housekeeping gene Gapdh or Hprt and presented by $2^{-\triangle \mathrm{Ct} 52}$.

Western blotting was performed using 4-20\% Mini-PROTEAN® TGX ${ }^{\mathrm{TM}}$ precast protein gels and the Trans-Blot ${ }^{\circledR}$ Turbo ${ }^{\mathrm{TM}}$ transfer system (Bio-Rad). The following antibodies: antiCHMP5 polyclonal antibody ${ }^{6}$, total OXPHOS antibody cocktail (Abcam), anti-GAPDH 
monoclonal antibody (GeneTex), and anti-beta-actin monoclonal antibody (Biolegend) were used.

\section{Extracellular Vesicle Tracking}

The concentration and size distribution of extracellular vesicles were analyzed using the NanoSight NS300 following manufacturer's protocol (Malvern Instruments). Briefly, cells were plated in 24-well plate at the density of $1 \times 10^{5}$ cells per well and cultured overnight. In the next morning, cells were changed to serum-free medium and incubated for $9 \mathrm{hrs}$. Medium was collected and subject to sequential centrifugation at $300 \mathrm{~g}$ for $10 \mathrm{~min}, 2000 \mathrm{~g}$ for $20 \mathrm{~min}$, and $10000 \mathrm{~g}$ for $30 \mathrm{~min}$. The resulted supernatant was manually injected into the instrument and run at the "standard measurement" module with five captures per sample and data were processed with a detection threshold of 2 .

\section{Live-cell Imaging for Cell Endocytosis}

Wild-type and Chmp5 $5^{\text {Ctsk }}$ skeletal progenitors cultured in MatTek glass bottom dishes were incubated on ice for $10 \mathrm{~min}$ before adding fresh medium with $2 \mathrm{ug} / \mathrm{ml} \mathrm{pHrodo}{ }^{\mathrm{TM}} \mathrm{red}$ EGF conjugate (ThermoFisher). Cells were incubated at $37^{\circ} \mathrm{C}$ for $30 \mathrm{~min}$ and subsequently washed and changed to fresh media. Live-cell images were obtained at 1, 12, and $24 \mathrm{hrs}$ using the Leica TCS SP5 II laser scanning confocal microscope.

\section{Transmission Electron Microscopy}

Wild-type and Chmp $5^{\text {Ctsk }}$ skeletal progenitors were fixed in culture plates overnight at $4{ }^{\circ} \mathrm{C}$ using $2.5 \%$ glutaraldehyde in $0.1 \mathrm{M} \mathrm{Na}$ cacodylate-HCl buffer (pH 7.2). After washing in $0.5 \mathrm{M}$ 
$\mathrm{Na}$ cacodylate-HCl buffer ( $\mathrm{pH} 7.0$ ), the cells were post-fixed for $1 \mathrm{hr}$ in $1 \%$ osmium tetroxide $(\mathrm{w} / \mathrm{v})$ at room temperature. Following post-fixation, the culture plate with adherent cells were enblock stained (20 min) with $1 \%$ aqueous uranyl-acetate $(\mathrm{w} / \mathrm{v})$. The culture plates were washed again in the same buffer and dehydrated through a graded series of ethanol to $100 \%$ and transferred through two changes of 50/50 (v/v) SPIpon resin (Structure Probe, Inc.)/100\% ethanol and left overnight to infiltrate. The following morning the cell culture plates were transferred through three changes of fresh SPIpon resin to finish the infiltration and embedding and finally, the plates were filled with freshly prepared SPIpon resin and polymerized for $48 \mathrm{hrs}$ at $70{ }^{\circ} \mathrm{C}$. Once fully polymerized, the plate was cut apart and each well was plunged into liquid nitrogen to separate the SPIpon epoxy block with the embedded cells from the culture dish. The round epoxy disks with the embedded cells were then examined under an upright light microscope and areas of cells were cut from the disks and glued onto blank microtome studs and trimmed for ultramicrotomy. Ultrathin sections $(70 \mathrm{~nm})$ were cut on a Reichart-Jung ultramicrotome using a diamond knife. The sections were collected and mounted on copper support grids and contrasted with lead citrate and uranyl acetate and examined on a FEI Tecnai G2 Spirit transmission electron microscope at $100 \mathrm{Kv}$ accelerating voltage and images were recorded at various magnifications using a Gatan $2 \mathrm{~K}$ digital camera system.

\section{Mitochondrial Respiration}

Mitochondrial respiration was examined in $C h m p 5^{\text {Ctsk }}$ and wild-type skeletal progenitors using a Seahorse $\mathrm{XF}^{\mathrm{e}} 96$ Extracellular Flux Analyzer (Agilent) as previously described ${ }^{53}$. Briefly, 10000 cells per well were plated in XF 96-well culture plates and cultured overnight. On the next day, cells were changed to assay medium and incubated at $37^{\circ} \mathrm{C}$ w/o CO2 for $1 \mathrm{hr}$. Assay was carried 
out using the Seahorse XF Cell Mito Stress Test kit with sequential injections of Oligomycin $(1 \mu \mathrm{M}), \mathrm{FCCP}(1 \mu \mathrm{M})$, and Rotenone plus Antimycin A $(0.5 \mu \mathrm{M})$ following the manufacturer's protocol.

\section{Tests of Skeletal Muscle Functions}

The four limb-hanging test was performed using the top grid of a mouse cage ${ }^{54}$. The grid was set at a height of about $35 \mathrm{~cm}$, and the measurement was repeated for 3-5 times for each mouse at a rest interval of about 3-5 min. The maximum hang time was recorded for each animal. Both male and female animals were included in this experiment and there was no significant sex difference regarding the four limb-hanging time.

The forelimb grip strength test was carried out using an electronic scale as described with slight modification ${ }^{54,55}$. A mouse was allowed to grasp the gauze attached on the scale. The scale was reset to $0 \mathrm{~g}$ after stabilization, and the mouse's tail was slowly pulled backward by a researcher. Five consecutive measurements were performed for each mouse and the peak pull force in grams was recorded. A significant sex difference was noticed in the forelimb grip strength. Both male and female mice were included in this study and the same trend of changes was found in both genders.

\section{Anti-resorptive, Antioxidant, and Anti-senescence Treatments}

Zolendronic acid monohydrate $(0.1 \mu \mathrm{g} / \mathrm{g}$ body weight, Sigma-Aldrich), OPG-Fc ( $3 \mu \mathrm{g} / \mathrm{g}$ body weight, Amgen Inc.), or PBS was intraperitoneally injected weekly to $C h m p 5^{f l f l}$ and $C h m p 5^{C t s k}$ 
mice starting at postnatal $\mathrm{d} 3$, and mice were sacrificed at 2, 4, and 6 weeks of ages for skeletal analysis.

Two doses of NAC (100 or $200 \mu \mathrm{g} / \mathrm{g}$ body weight) were used for the antioxidant treatment starting from the first week after birth. Animals were monitored twice per week. The symptoms of Chmp5 $5^{\text {Ctsk }}$ mice became more severe with the treatment, while $C h m p 5^{\text {Ctsk/+ }}$ and $C h m p 5^{\text {fllfl }}$ animals did not display gross abnormalities.

Senolytic drugs quercetin and dasatinib were intraperitoneally administrated weekly at $50 \mu \mathrm{g} / \mathrm{g}$ and $5 \mu \mathrm{g} / \mathrm{g}$ body weight respectively. For $C h m p 5^{\text {Ctsk }}$ strain, the anti-senescence treatment started from the first week after birth and mice were collected at 7-8 weeks of age for skeletal analyses. For Chmp $5^{\text {Dmp } 1}$ strain, treatment began from the second week after birth and animals were monitored for 16 weeks. An improvement of animal motility was also observed with the treatment in adult mice. Increased drug dose (twice per week instead of weekly) could cause side-effects such as inflammation in eyes.

\section{Statistics}

All data are graphically represented as the mean \pm s.d.. For experiments with three or more groups, statistical analysis was performed using one-way or two-way ANOVA followed by Sidak's correction for multiple comparisons. For two-group comparisons, the two-tailed, unpaired Student's $t$-test was applied. All analyses were performed using Prism 8.0 (GraphPad). 


\section{References}

1. Kaksonen, M. \& Roux, A. Mechanisms of clathrin-mediated endocytosis. Nat Rev Mol Cell Biol 19, 313-326 (2018).

2. Christ, L., Raiborg, C., Wenzel, E.M., Campsteijn, C. \& Stenmark, H. Cellular Functions and Molecular Mechanisms of the ESCRT Membrane-Scission Machinery. Trends Biochem Sci 42, 42-56 (2017).

3. Luzio, J.P., Pryor, P.R. \& Bright, N.A. Lysosomes: fusion and function. Nat Rev Mol Cell Biol 8, 622-632 (2007).

4. Hurley, J.H. ESCRTs are everywhere. EMBO J 34, 2398-2407 (2015).

5. Schmidt, O. \& Teis, D. The ESCRT machinery. Curr Biol 22, R116-120 (2012).

6. Shim, J.H. et al. CHMP5 is essential for late endosome function and down-regulation of receptor signaling during mouse embryogenesis. J Cell Biol 172, 1045-1056 (2006).

7. Kranz, A., Kinner, A. \& Kolling, R. A family of small coiled-coil-forming proteins functioning at the late endosome in yeast. Mol Biol Cell 12, 711-723 (2001).

8. Adoro, S. et al. Post-translational control of T cell development by the ESCRT protein CHMP5. Nat Immunol 18, 780-790 (2017).

9. Greenblatt, M.B. et al. CHMP5 controls bone turnover rates by dampening NF-kappaB activity in osteoclasts. $J$ Exp Med 212, 1283-1301 (2015).

10. Das, A., Nag, S., Mason, A.B. \& Barroso, M.M. Endosome-mitochondria interactions are modulated by iron release from transferrin. J Cell Biol 214, 831-845 (2016).

11. Chen, M. et al. Extracellular anti-angiogenic proteins augment an endosomal protein trafficking pathway to reach mitochondria and execute apoptosis in HUVECs. Cell Death Differ 25, 1905-1920 (2018).

12. Farmer, T. et al. Control of mitochondrial homeostasis by endocytic regulatory proteins. J Cell Sci 130, 2359-2370 (2017).

13. Wong, Y.C., Ysselstein, D. \& Krainc, D. Mitochondria-lysosome contacts regulate mitochondrial fission via RAB7 GTP hydrolysis. Nature 554, 382-386 (2018).

14. Sugiura, A., McLelland, G.L., Fon, E.A. \& McBride, H.M. A new pathway for mitochondrial quality control: mitochondrial-derived vesicles. EMBO J 33, 2142-2156 (2014).

15. Hammerling, B.C. et al. A Rab5 endosomal pathway mediates Parkin-dependent mitochondrial clearance. Nat Commun 8, 14050 (2017).

16. Yang, W. et al. Ptpn11 deletion in a novel progenitor causes metachondromatosis by inducing hedgehog signalling. Nature 499, 491-495 (2013).

17. Debnath, S. et al. Discovery of a periosteal stem cell mediating intramembranous bone formation. Nature 562, 133-139 (2018).

18. Shi, R. et al. A migratory stem cell population for musculoskeletal enveloping tissues and the bone. SSRN 3281656, 2018 (2018).

19. Seifert, W. et al. HPGD mutations cause cranioosteoarthropathy but not autosomal dominant digital clubbing. Eur J Hum Genet 17, 1570-1576 (2009).

20. Dabir, T. et al. Cranio-osteoarthropathy in sibs. Clin Dysmorphol 16, 197-201 (2007).

21. Leussink, B. et al. Expression patterns of the paired-related homeobox genes MHox/Prx1 and S8/Prx2 suggest roles in development of the heart and the forebrain. Mech Dev 52, 51-64 (1995). 
22. Ono, N. et al. Vasculature-associated cells expressing nestin in developing bones encompass early cells in the osteoblast and endothelial lineage. Dev Cell 29, 330-339 (2014).

23. Maes, C. et al. Osteoblast precursors, but not mature osteoblasts, move into developing and fractured bones along with invading blood vessels. Dev Cell 19, 329-344 (2010).

24. Lim, J. et al. Dual function of Bmprla signaling in restricting preosteoblast proliferation and stimulating osteoblast activity in mouse. Development 143, 339-347 (2016).

25. Lim, J., Burclaff, J., He, G., Mills, J.C. \& Long, F. Unintended targeting of Dmp1-Cre reveals a critical role for Bmprla signaling in the gastrointestinal mesenchyme of adult mice. Bone Res 5, 16049 (2017).

26. Ge, X. et al. NFAT restricts osteochondroma formation from entheseal progenitors. JCI Insight 1, e86254 (2016).

27. Chapman, J., Fielder, E. \& Passos, J.F. Mitochondrial dysfunction and cell senescence: deciphering a complex relationship. FEBS Lett 593, 1566-1579 (2019).

28. Gorgoulis, V. et al. Cellular Senescence: Defining a Path Forward. Cell 179, 813-827 (2019).

29. Korolchuk, V.I., Miwa, S., Carroll, B. \& von Zglinicki, T. Mitochondria in Cell Senescence: Is Mitophagy the Weakest Link? EBioMedicine 21, 7-13 (2017).

30. Birch, J. \& Passos, J.F. Targeting the SASP to combat ageing: Mitochondria as possible intracellular allies? Bioessays 39 (2017).

31. Vild, C.J., Li, Y., Guo, E.Z., Liu, Y. \& Xu, Z. A novel mechanism of regulating the ATPase VPS4 by its cofactor LIP5 and the endosomal sorting complex required for transport (ESCRT)-III protein CHMP5. J Biol Chem 290, 7291-7303 (2015).

32. Yang, Z. et al. Structural basis of molecular recognition between ESCRT-III-like protein Vps60 and AAA-ATPase regulator Vta1 in the multivesicular body pathway. J Biol Chem 287, 43899-43908 (2012).

33. Kenyon, K.R., Sensenbrenner, J.A. \& Wyllie, R.G. Hepatic ultrastructure and histochemistry in mucolipidosis II (I-cell disease). Pediatr Res 7, 560-568 (1973).

34. Abe, K. et al. Ultrastructural studies in fetal I-cell disease. Pediatr Res 10, 669-676 (1976).

35. Saftig, P. \& Klumperman, J. Lysosome biogenesis and lysosomal membrane proteins: trafficking meets function. Nat Rev Mol Cell Biol 10, 623-635 (2009).

36. Todkar, K., Ilamathi, H.S. \& Germain, M. Mitochondria and Lysosomes: Discovering Bonds. Front Cell Dev Biol 5, 106 (2017).

37. Plotegher, N. \& Duchen, M.R. Mitochondrial Dysfunction and Neurodegeneration in Lysosomal Storage Disorders. Trends Mol Med 23, 116-134 (2017).

38. Rappaport, J., Manthe, R.L., Solomon, M., Garnacho, C. \& Muro, S. A Comparative Study on the Alterations of Endocytic Pathways in Multiple Lysosomal Storage Disorders. Mol Pharm 13, 357-368 (2016).

39. Herz, H.M. et al. vps25 mosaics display non-autonomous cell survival and overgrowth, and autonomous apoptosis. Development 133, 1871-1880 (2006).

40. Vaccari, T. \& Bilder, D. The Drosophila tumor suppressor vps 25 prevents nonautonomous overproliferation by regulating notch trafficking. Dev Cell 9, 687-698 (2005). 
41. Moberg, K.H., Schelble, S., Burdick, S.K. \& Hariharan, I.K. Mutations in erupted, the Drosophila ortholog of mammalian tumor susceptibility gene 101, elicit non-cellautonomous overgrowth. Dev Cell 9, 699-710 (2005).

42. Raposo, G. \& Stoorvogel, W. Extracellular vesicles: exosomes, microvesicles, and friends. J Cell Biol 200, 373-383 (2013).

43. Coly, P.M. \& Boulanger, C.M. Extracellular Mitochondria and Vesicles. Circ Res 125, 53-54 (2019).

44. Luzio, J.P., Hackmann, Y., Dieckmann, N.M. \& Griffiths, G.M. The biogenesis of lysosomes and lysosome-related organelles. Cold Spring Harb Perspect Biol 6, a016840 (2014).

45. Lu, Y. et al. DMP1-targeted Cre expression in odontoblasts and osteocytes. J Dent Res 86, 320-325 (2007).

46. Rodda, S.J. \& McMahon, A.P. Distinct roles for Hedgehog and canonical Wnt signaling in specification, differentiation and maintenance of osteoblast progenitors. Development 133, 3231-3244 (2006).

47. Logan, M. et al. Expression of Cre Recombinase in the developing mouse limb bud driven by a Prxl enhancer. Genesis 33, 77-80 (2002).

48. Langmead, B. \& Salzberg, S.L. Fast gapped-read alignment with Bowtie 2. Nat Methods 9, 357-359 (2012).

49. Dobin, A. et al. STAR: ultrafast universal RNA-seq aligner. Bioinformatics 29, 15-21 (2013).

50. Trapnell, C. et al. Differential analysis of gene regulation at transcript resolution with RNA-seq. Nat Biotechnol 31, 46-53 (2013).

51. Fabregat, A. et al. Reactome pathway analysis: a high-performance in-memory approach. BMC Bioinformatics 18, 142 (2017).

52. Schmittgen, T.D. \& Livak, K.J. Analyzing real-time PCR data by the comparative C(T) method. Nat Protoc 3, 1101-1108 (2008).

53. Fiorese, C.J. et al. The Transcription Factor ATF5 Mediates a Mammalian Mitochondrial UPR. Curr Biol 26, 2037-2043 (2016).

54. Bonetto, A., Andersson, D.C. \& Waning, D.L. Assessment of muscle mass and strength in mice. Bonekey Rep 4, 732 (2015).

55. Takeshita, H. et al. Modified forelimb grip strength test detects aging-associated physiological decline in skeletal muscle function in male mice. Sci Rep 7, 42323 (2017). 


\section{Figure Legends}

\section{Fig. 1: Ablation of Chmp5 in Ctsk-expressing skeletal progenitors causes aberrant bone} growth.

a Micro-CT and Haemotoxylin and Eosin (H\&E) staining showing periskeletal overgrowth near the joint in $C h m p 5^{C t s k}$ mice in comparison with $C h m p 5^{f l f l}$ mice at 7 weeks of age. Dot-line representing the approximate border between the periskeletal overgrowth and bone at the femoral condyle of the knee. Images are representative of 10 animals per group. b Micro-CT images displaying bone overgrowth at the ankle and foot in $C h m p 5^{\text {Ctsk }}$ mice at one year of age. Images are representative of 4 animals per group. c Micro-CT, TRAP staining, and measurement of orbital distance and head width demonstrating craniofacial suture abnormalities in Chmp $5^{\text {Ctsk }}$ mice at 6 weeks of age. Images are representative of $10 \mathrm{Chmp5}^{f l f l}$ and $11 C h m p 5^{\text {Ctsk }}$ mice. d, e Confocal images mapping $\mathrm{Ctsk}^{+}\left(\mathrm{GFP}^{+}\right)$progenitors and their descendants in periskeletal tissues (d) and the sagittal suture (e) in $C h m p 5^{C t s k /+} ; \operatorname{Rosa} 26^{m T m G /+}$ and $C h m p 5^{C t s k} ; \operatorname{Rosa} 26^{m T m G /+}$ mice at 2 weeks of age. In (d), asterisk indicating periskeletal overgrowths; dot-line representing the approximate border between the periskeletal overgrowth and bone. Images are representative of 4 animals each group. f Flow cytometry showing strategy of sorting $\mathrm{CD}^{-} 5^{-} \mathrm{CD}^{-} 1^{-} \mathrm{GFP}^{+}$and $\mathrm{CD}^{-} 5^{-} \mathrm{CD}^{-} 1^{-}$; $\mathrm{GFP}^{-}$skeletal progenitors from periskeletal tissues of $\mathrm{Chmp5}^{\mathrm{Ctsk/+}} ; \mathrm{Rosa}^{\mathrm{mTmG/+}}$ and $C h m p 5^{\text {Ctsk }} ; \operatorname{Rosa} 26^{m T m G /+}$ mice at 2 weeks of age. g Quantitative PCR determining the deletion of Chmp5 in $C h m p 5^{\text {Ctsk }}$ relative to $C h m p 5^{\text {Ctsk/+ }}$ skeletal progenitors. $n=6$ mice per group. h Quantitative PCR determining the expression of PGE2 pathway related genes Hpgd, Ptgs 1, Ptgs 2, and Abcc4 in $C h m p 5^{C t s k}$ relative to $C h m p 5^{C t s k /+}$ skeletal progenitors. $n=6$ animals per group. All data are mean \pm s.d.; two-tailed Student's $t$-test. Scale bars, $200 \mu \mathrm{m}$ except $1 \mathrm{~mm}$ for micro-CT images. 


\section{Fig. 2: Ablation of Chmp5 in Dmpl-expressing progenitors causes abnormal bone} expansion and myopathy.

a X-ray images demonstrating progressive cortical bone expansion (arrows) and decreased skeletal muscle volume (asterisks) in $C h m p 5^{D m p l}$ mice in comparison with $C h m p 5^{f l f l}$ mice. Images are representative of 3, 3, 10, 8 animals per group for 3 weeks, 5 weeks, 10 weeks, and 1 year of ages respectively. Scale bars, $10 \mathrm{~mm}$. b Gross image and measurement of femur thickness showing bone expansion in $C h m p 5^{D m p l}$ versus $C h m p 5^{f l f l}$ mice at 10 weeks of age. $n=$ $13 \mathrm{Chmp}^{\text {fllfl }}$ and $12 \mathrm{Chmp}^{\mathrm{Dmpl}}$ mice. Scale bar, $1 \mathrm{~mm}$. c Micro-CT analysis displaying cortical bone expansion and decreased trabecular bone mass in $C h m p 5^{D m p l}$ relative to $C h m p 5^{f l f l}$ mice at 10 weeks of age. $n=6 C h m p 5^{f l / f l}$ and $5 C h m p 5^{D m p l}$ male mice, similar trend of changes found in both genders. Scale bars, $1 \mathrm{~mm}$. d H\&E staining showing bone expansion at the endosteum (arrows) and decreased trabecular bone mass (right) in $C h m p 5^{D m p 1}$ mice. The left panels showing midshaft and the right panels showing metaphysis of the femur bone. Images are representative of 3 mice per group. Scale bars, $0.5 \mathrm{~mm}$. e Micro-CT images demonstrating craniofacial bone expansion (arrows) in $C h m p 5^{D m p l}$ relative to $C h m p 5^{f l / f l}$ mice at 10 weeks of age. $n=6 C h m p 5^{f l / f l}$ and $5 C h m p 5^{D m p l}$ animals. f Hindlimb abduction of $C h m p 5^{D m p l}$ mice in comparison with $C h m p 5^{f l f l}$ littermate controls at 10 weeks of age. More than 20 animals examined for each group. g Skeletal muscle mass (quadriceps and gastrocnemius, $n=4$ per group, male mice), four-limb handing time ( $n=10$ per group, pooled data from male and female mice), and forelimb pull strength ( $n=6$ each group, male mice) in $C h m p 5^{D m p 1}$ mice compared to $C h m p 5^{f l f l}$ mice at 10-12 weeks of age. For skeletal muscle mass and forelimb pull strength, similar trend of changes was 
found in both genders. All data are mean \pm s.d.; two-tailed Student's $t$-test for 2-group comparison or 2way ANOVA followed by Sidak's test for multiple comparisons.

Fig. 3: Chmp5 deficiency induces cellular senescence and increases osteogenic capacity of skeletal progenitors.

a Cell number counting and AlamarBlue assay determining cell proliferation in $C h m p 5^{\text {Ctsk }}$ and Chmp $5^{\text {Ctsk/+ }}$ skeletal progenitors at 2 weeks of age. $n=3$ replicates per group per time-point; experiments repeated 3 times using cells from 3 mice. b Gene set enrichment analysis (GSEA) of transcriptome data showing positive enrichment of genes associated with cell senescence in Chmp $5^{\text {Ctsk }}$ relative to $C h m p 5^{\text {Ctsk/+ }}$ skeletal progenitors. Enriched gene list referring to

Supplementary Table 1. $n=3$ mice per group for RNA-sequencing analysis. c Gross image demonstrating phenotypes of accelerating senescence in $C h m p 5^{C t s k}$ mice compared with Chmp5 $5^{\text {Ctsk/+ }}$ mice at one year of age. Images are representative of 5 animals per group. d Cell number counting determining cell proliferation in ATDC5 cells with or without Chmp5 deletion by lentiviral CRISPR/CAS9. $n=4$ replicates per group per time-point, experiment repeated three times. e Cell cycle analysis in ATDC5 cells with or without Chmp5 deletion. $n=3$ replicates per group, results repeated twice. f Alizarin red staining, von Kossa staining and alkaline phosphatase activity assay determining osteogenic activity in Chmp5 $5^{\text {Ctsk }}$ compared to Chmp $5^{\text {Ctsk/+ }}$ skeletal progenitors. $n=3$ replicates each group, representative results of 3 mice per group. $\mathbf{g}$ Ingenuity pathway analysis of transcriptome data showing increased activity of osteoblast differentiation-related pathways in $C h m p 5^{\text {Ctsk }}$ compared to Chmp $5^{\text {Ctsk/+ }}$ skeletal progenitors. Gene lists seeing Supplementary Table 2. h Alizarin red staining and AlarmaBlue assay demonstrating osteogenesis and proliferation activity respectively in MC3T3-E1 cells with or 
without Chmp5 deletion by lentiviral CRISPR/CAS9. $n=4$ replicates per group for alizarin red staining, $n=12$ per group for AlarmaBlue assay; experiments repeated twice. All data are mean \pm s.d.; two-tailed Student's $t$-test for 2-group comparison or 2way ANOVA followed by Sidak's test for multiple comparisons.

\section{Fig. 4: Paracrine actions of Chmp5-deficient skeletal progenitors.}

a GSEA of transcriptome data showing positive enrichment of genes associated with the senescence-associated secretory phenotype in $C h m p 5^{\text {Ctsk }}$ relative to $C h m p 5^{\text {Ctsk/+ }}$ skeletal progenitors. Enriched gene list referring to Supplementary Table 1. b Confocal images demonstrating extracellular vesicles (arrowheads) around Chmp $5^{\text {Ctsk }}$ skeletal progenitors. Images are representative of 30 cells from 3 animals per group. Scale bars, $10 \mu \mathrm{m}$. c-e Nanoparticle tracking analysis of extracellular vesicles in culture media of Chmp $5^{\text {Ctsk }}$ and wild-type skeletal progenitors. Data pooled from 3 replicates per group, 5 reads for each; repeated twice using cells from 2 mice per group. f Cell number counting determining cell proliferation in ATDC5 cells after treatment with culture supernatant from Chmp5-deficient or Chmp5-sufficient cells. $n=3$ replicates per group per time-point, repeated twice. $\mathbf{g}, \mathbf{h}$ Cell number counting, AlarmaBlue assay, and alizarin red staining analyzing cell proliferation (g) and osteogenesis (h) in neighboring $\mathrm{CD} 45^{-}$; $\mathrm{CD}^{-} 1^{-}$; $\mathrm{GFP}^{-}$progenitors sorted from $\mathrm{Chmp}^{\text {Ctsk }} ; \mathrm{Rosa}_{26^{m T m G /+}}$ or CtskCre;Rosa $26^{m T m G /+}$ mice. $n=3$ replicates per group per time-point for cell number counting and alizarin red staining, $n=12$ replicates per group for AlarmaBlue assay; repeated 3 times using cells from 3 animals. i Immunostaining of cell proliferation marker Ki-67 in periskeletal tissues around the knee of $C h m p 5^{\text {Ctsk }}$ and $C h m p 5^{\text {Ctsk/+ }}$ mice. $n=3$ animals per group; dot-line representing the approximate border between the periskeletal overgrowth and femoral condyle; 
scale bars, $100 \mu \mathrm{m}$. All data are mean \pm s.d.; two-tailed Student's $t$-test for 2-group comparison or 2way ANOVA followed by Sidak's test for multiple comparisons.

Fig. 5: Chmp5 deficiency perturbs the endocytic pathway in skeletal progenitors.

a Representative confocal images demonstrating abnormally enlarged vesicles in $C h m p 5^{\text {Ctsk }}$ relative to wild-type skeletal progenitors. Abnormal cells identified by containing enlarged GFP ${ }^{+}$ vesicles. $n=200$ cells from three mice per group. b Schematic showing molecular markers utilized for analyzing the endocytic pathway. c Representative confocal images showing LAMP1 immunostaining in Chmp $5^{\text {Ctsk }}$ and wild-type skeletal progenitors. $n=20$ cells each group. d Quantification of $\mathrm{LAMP}^{+}$vesicles in ATDC5 cells with or without Chmp5 depletion. $n=93,66$ cells respectively. e Representative confocal images showing RAB7 immunostaining in Chmp5 ${ }^{\text {Ctsk }}$ and wild-type skeletal progenitors. $n=15$ cells per genotype. f Quantification of $\mathrm{RAB}^{+}$vesicles in ATDC5 cells with or without Chmp5 depletion. $n=94,92$ cells respectively. g Co-localization of LAMP1 and RAB7 in ATDC5 cells with or without Chmp5 depletion. $n=$ 20 cells per group. $\mathbf{h}$ Transmission electron microscopy (TEM) showing accumulation of multivesicular body (MVB)-like (arrows) and lysosome-like (arrowheads) structures in Chmp $5^{\text {Ctsk }}$ relative to wild-type skeletal progenitors. $n=30$ cells per group. i Confocal live-cell images demonstrating delayed degradation of EGF-conjugate in $C h m p 5^{\text {Ctsk }}$ versus wild-type skeletal progenitors. $n=10$ cells each group per time-point. Data shown as mean \pm s.d.; twotailed Student's $t$-test. Scale bars, $10 \mu \mathrm{m}$ except in (h) as indicated.

Fig. 6: Chmp5 deficiency causes mitochondrial dysfunction to induce cell senescence 
a GSEA of transcriptome data reporting positive enrichment of genes associated with oxidative stress-induced senescence in $C h m p 5^{\text {Ctsk }}$ relative to Chmp5 $5^{\text {Ctsk/+ }}$ skeletal progenitors. Enriched gene list referring to Supplementary Table 1. b Confocal fluorescence imaging showing intracellular ROS (indicated by CellROX Deep Red) and mitochondria (indicated by TMRE) in Chmp $5^{\text {Ctsk }}$ versus wild-type skeletal progenitors. Images are representative of 30 cells per group. Scale bars, $15 \mu \mathrm{m}$. c Quantification of cellular oxidative stress by examining fluorescence intensity of CellROX Deep Red. $n=10$ each group, results repeated twice. $\mathbf{d}$ Confocal fluorescence imaging mapping intracellular ROS (indicated by CellROX Deep Red) and mitochondria (indicated by MitoTracker Green) in ATDC5 cells with or without Chmp5 depletion. $n=20$ cells each group; scale bars, $10 \mu \mathrm{m}$. e Flow cytometry quantifying CellROX Deep Red stain in ATDC5 cells with or without Chmp5 depletion. $n=3$ per group, experiment repeated twice. f Quantification of TMRE fluorescence intensity in Chmp5-sufficient and Chmp5-deficient ATDC5 cells. $n=12$ each group, repeated 3 times. g Western blotting demonstrating changes of mitochondrial OXPHOS proteins NDUF88, SDHB, MT-CO1, UQCRC2, and ATF5A in Chmp5-deficient relative to Chmp5-sufficient ATDC5 cells. Experiment repeated twice. $\mathbf{h}$ Cell number counting determining cell proliferation in galactose medium. The same number of cells was plated at day 0 and cultured for 7 days. $n=6$ per group, repeated three times. i Seahorse cell mitochondrial stress test showing mitochondrial respiratory capacity and extracellular acidification rate in $C h m p 5^{\text {Ctsk }}$ relative to wild-type skeletal progenitors. Data pooled from cells of 3 mice, 8 replicates for each animal. $\mathbf{j}$ TEM images showing mitochondrial morphology in $C h m p 5^{\text {Ctsk }}$ versus wild-type skeletal progenitors. $n=50$ cells each group; scale bars, $200 \mathrm{~nm}$. k GSEA of transcriptome data and qPCR demonstrating positive enrichment of genes associated with DNA damage-induced senescence and upregulation 
of DNA damage responsive genes $H 2 a f x$ and $T r p 53$ respectively in $C h m p 5^{\text {Ctsk }}$ relative to Chmp $5^{\text {Ctsk/+ }}$ skeletal progenitors. $n=3$ mice per group for RNA-sequencing analysis; $n=6$ animals per group for qPCR. Enriched gene list for GSEA referring to Supplementary Table 1. All data shown as mean \pm s.d.; two-tailed Student's $t$-test.

\section{Fig. 7: Targeting senescent cells using senolytic drugs mitigates bone overgrowth and} myopathy in $\mathrm{Chmp5}^{\text {Ctsk }}$ and $\mathrm{Chmp5}^{\mathrm{Dmp1}}$ mice.

a Cell number counting and AlamarBlue assay determining cell proliferation in ATDC5 cells with or without Chmp5 depletion after treatment with the antioxidant $N$-Acetylcysteine (NAC). $n$ $=3$ or 12 each group for cell number counting or AlamarBlue assay respectively, results repeated 3 times. b Alizarin red staining showing mineralization activity after treatment with NAC in Chmp $5^{\text {Ctsk }}$ versus wild-type skeletal progenitors under osteogenic induction for 3 weeks. $n=3$ or 4 per group, result repeated 5 times. c Gross images and radiography demonstrating hindlimb abduction and periskeletal bone overgrowth (arrows) respectively in $C h m p 5^{\text {Ctsk }}$ mice in comparison with $C h m p 5^{f l f l}$ mice after treatment with quercetin and dasatinib $(\mathrm{Q}+\mathrm{D})$ or the vehicle PEG400 weekly for 7 weeks. $n=8$-10 mice per group. d, e Measurement of mouthopening and micro-CT images showing the improvement of the temporomandibular joint lesion in $C h m p 5^{\text {Ctsk }}$ mice after treatment with $\mathrm{Q}+\mathrm{D}$. Arrows indicating bone overgrowth around the mandibular condyle. Scale bars, $1 \mathrm{~mm}$. f Animal body weight after treatment with Q + D or the vehicle PEG400. $n=5$ mice per group; data of female mice shown; similar results found in both genders. g Four-limb hanging time and forelimb pull strength in $C h m p 5^{D m p l}$ and $C h m p 5^{f l f l}$ mice after treatment with $\mathrm{Q}+\mathrm{D}$ or the vehicle PEG400. $n=12$ animals per group from both genders for hanging time test, 7 male mice per group for forelimb pull strength test; similar trend of 
bioRxiv preprint doi: https://doi.org/10.1101/2020.08.03.233874; this version posted August 4, 2020. The copyright holder for this preprint (which was not certified by peer review) is the author/funder. All rights reserved. No reuse allowed without permission.

changes found in both genders for the forelimb pull strength. All data are mean \pm s.d.; 2 way ANOVA followed by Sidak's test for multiple comparisons. 


\section{Supplementary Figure Legends}

\section{Supplementary Fig. 1 Deletion of Chmp5 in $\mathrm{Ctsk}^{+}$skeletal progenitors, not osteoclasts, is responsible for periskeletal and suture lesions in $\operatorname{Chmp5}^{\mathrm{Ctsk}}$ mice.}

a Micro-CT images showing progressive craniofacial suture and zygomatic bone (arrows) lesions with age. Images are representative of 6 mice per group at 7 weeks of age, 5 mice per group at 18 mons of age. b Representative micro-CT images displaying abnormal bone formation around the mandibular condyle of temporomandibular joint (arrow) with limited mouth-opening. $n=37 C h m p 5^{f l / f l}, 33 C h m p 5^{C t s k}$ mice. c TRAP staining showing no osteoclasts in the periskeletal overgrowth (asterisk) in $C h m p 5^{C t s k}$ mice at 2 weeks of age. TRAP ${ }^{+}$osteoclasts in the bone marrow as the positive control; dot-line representing the approximate border between the periskeletal overgrowth and bone at the femoral condyle of the knee. Images are representative of 3 animals per group. $\mathbf{d}$-h Gross and micro-CT images demonstrating skeletal phenotypes after treatment with PBS, Zoledronic acid, or OPG-Fc at 2 weeks (d-f) or 4 weeks (g, h) of ages. Both arrows and arrowheads indicating periskeletal overgrowths. Images are representative of $4(\mathbf{d}), 5(\mathbf{e}, \mathbf{f})$, or $3(\mathbf{g}, \mathbf{h})$ mice per group. i Confocal images of Rosa26 $6^{m T m G /+}$ $\left(\mathrm{Cre}^{-}\right)$control mice. Related to Fig. 1d, e. j Unstained cells from Rosa2 $6^{m T m G /+}$ mice as a negative control for sorting $\mathrm{Ctsk}^{+}$skeletal progenitors. Related to Fig. 1f. Data are mean \pm s.d.; two-tailed Student's $t$-test. Scale bars, $1 \mathrm{~mm}$ for micro-CT images, $200 \mu \mathrm{m}$ for histological images. 


\section{Supplementary Fig. 2 Skeletal abnormalities after deleting Chmp5 in Dmp1 progenitors.}

a Representative fluorescence image showing fate-mapping of $\mathrm{Dmpl}^{+}\left(\mathrm{GFP}^{+}\right)$osteoprogenitors and their descendants in the tibia of Dmpl-Cre;Rosa $26^{m T m G /+}$ mice at 4 weeks of age. b, c Micro$\mathrm{CT}$ analyses of trabecular and cortical bone parameters in $C h m p 5^{D m p l}$ mice compared to Chmp $5^{f l f l}$ mice. $n=5$ animals per group, representative data from male mice shown, similar trend of changes found in both genders. $\mathbf{d}$ TRAP staining and activity assay determining osteoclastogenesis in bone marrow stromal cells of $C h m p 5^{D m p l}$ and $C h m p 5^{f l f l}$ mice. $n=4$ animals per group, repeated twice. Data shown as mean \pm s.d.; two-tailed Student's $t$-test for 2-group comparison or 2way ANOVA followed by Sidak's test for multiple comparisons. Scale bars, 200 $\mu \mathrm{m}$.

\section{Supplementary Fig. 3 Chmp5 deficiency induces cellular senescence and enhances} osteogenic capacity of skeletal progenitors.

a Cell number counting and AlamarBlue assay determining cell proliferation in $\mathrm{Ctsk}^{+}$suture progenitors isolated from $C h m p 5^{\text {Ctsk }} ; \operatorname{Rosa}_{2} 6^{m T m G /+}$ or $C h m p 5^{\text {Ctsk/+ }} ; \operatorname{Rosa} 26^{m T m G /+}$ mice at 2 weeks of age. $n=3$ replicates per group per time-point for cell number counting, $n=12$ replicates per group for Alamarblue assay; representative results of 3 animals for each group. b Colony formation assay for $\mathrm{Dmpl}^{+}$osteoprogenitors isolated from bones of $C h m p 5^{\mathrm{Dmpl}} ; \operatorname{Rosa} 26^{m T m G /+}$ or $C h m p 5^{\text {Dmp } 1 /+} ; \operatorname{Rosa} 26^{m T m G /+}$ mice at 3 weeks of age. $n=5$ animals per group. c TUNEL staining determining cell apoptosis in craniofacial sutures of $C h m p 5^{C t s k}$ and $C h m p 5^{C t s k /+}$ mice. $n=3$ animals per group; scale bars, $100 \mu \mathrm{m}$. d Annexin V stain examining cell apoptosis in Chmp $5^{\text {Ctsk }}$ and Chmp $5^{\text {Ctsk/+ }}$ skeletal progenitors. $n=3$ replicates each group; repeated twice using cells from 2 mice per group. e Western blotting showing deletion of Chmp5 in ATDC5 cells by lentiviral 
CRISPR/CAS9. $\mathbf{f}$ AlamarBlue assay determining cell proliferation in ATDC5 cells with or without Chmp5 deletion. $n=12$ replicates per group, repeated 3 times. g Annexin V-PI stain analyzing cell apoptosis in ATDC5 cells with or without Chmp5 deletion. $n=3-5$ replicates each group, repeated 3 times. $\mathbf{h}$ Cell number counting examining cell proliferation in ATDC5 cells with or without Chmp5 deletion after treatment with the pan-caspase inhibitor Q-VD-OPh. $n=3$ replicates each group each dose, repeated twice. i Quantitative PCR determining expression of Col2al and Sox 9 in $C h m p 5^{\text {Ctsk }}$ and $C h m p 5^{\text {Ctsk/+ }}$ skeletal progenitors. $n=6$ mice per group. $\mathbf{j}$ PANTHER GO-BP over-representation analysis of transcriptome data showing decreased

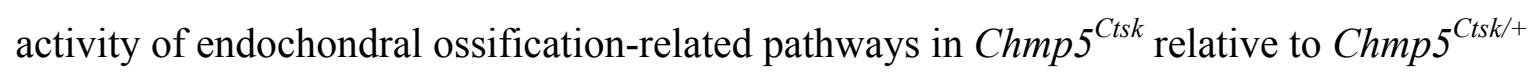
skeletal progenitors. Minus (-), under-represented terms; $n=3$ mice per group for RNAsequencing analysis. $\mathbf{k}$ Western blotting and alizarin red staining respectively showing deletion of Chmp5 in mouse MC3T3-E1 cells by lentiviral CRISPR/CAS9 and increased activity of mineralization in Chmp5-deficient versus Chmp5-sufficient MC3T3-E1 cells when induced toward osteogenesis. $n=4$ replicates per group, repeated twice. All data are mean \pm s.d.; 2 way ANOVA followed by Sidak's test for multiple comparisons or two-tailed Student's $t$-test for 2group comparison.

\section{Supplementary Fig. 4 Secretory phenotype of Chmp5-deficient skeletal progenitors.}

a Nanoparticle tracking analysis showing size distribution of extracellular vesicles in $C h m p 5^{\text {Ctsk }}$ and wild-type skeletal progenitors. Data pooled from 3 replicates, 5 reads for each; repeated twice using cells from 2 mice; D10, D50, and D90 indicating percent undersize, for example $\mathrm{D} 50=229 \mathrm{~nm}$ representing that 50\% vesicles are $229 \mathrm{~nm}$ or smaller. b, c Nanoparticle tracking analysis in ATDC5 cells with or without Chmp5 deletion. Data pooled from 2 replicates, 
5 reads for each; repeated twice. $\mathbf{d}$ AlamarBlue assay determining activity of cell proliferation in ATDC5 cells after treatment with conditioned medium from Chmp5-sufficient or Chmp5deficient cells. $n=12$ replicates each group, repeated 3 times. e Immunostaining of Ki-67 in proximal tibia of the knee in $C h m p 5^{\text {Ctsk }}$ versus $C h m p 5^{\text {Ctsk/+ }}$ mice. Dot-lines representing the approximate border between perichondrial tissues and the tibial growth-plate. $n=3$ animals per group; scale bars $100 \mu \mathrm{m}$. f Upregulated genes, coding secretory factors that potentially promote osteogenesis via a paracrine mechanism, in $C h m p 5^{\text {Ctsk }}$ relative to $C h m p 5^{\text {Ctsk/+ }}$ skeletal progenitors. $n=3$ mice per group for RNA-sequencing analysis. All data are mean \pm s.d.; twotailed Student's $t$-test for 2-group comparison or 2way ANOVA followed by Sidak's test for multiple comparisons.

\section{Supplementary Fig. 5 Aberrant endocytic pathway in Chmp5-deficient skeletal progenitors.}

a Representative confocal fluorescence images and quantification of fluorescence intensity of LysoTracker Red DND-99 in Chmp $5^{\text {Ctsk }}$ compared to wild-type skeletal progenitors. $n=11$ replicates per group for quantitative analysis; repeated 3 times using cells from 3 mice. Scale bars, $50 \mu \mathrm{m}$. b Isotype controls for immunofluorescence staining. Scale bars, $20 \mu \mathrm{m}$. c, d Confocal images of LAMP1(c) and RAB7 (d) immunostaining in ATDC5 cells with or without Chmp5 deletion. Scale bars, $20 \mu \mathrm{m}(\mathbf{c}), 10 \mu \mathrm{m}$ (d). e Additional TEM images showing accumulation of MVB-like (arrows) and lysosome-like (arrowheads) structures in Chmp5deficient skeletal progenitors. f-h Representative confocal images of immunostaining for early endosome marker EEA1 (f), cycling endosome marker RAB11 (g), and trans-Golgi network marker TGN38 (h) in Chmp5 ${ }^{\text {Ctsk }}$ versus wild-type skeletal progenitors. $n=30$ cells per group. 
Scale bars, $10 \mu \mathrm{m}$ (e), $20 \mu \mathrm{m}$ (f), $25 \mu \mathrm{m}$ (g). Data shown as mean \pm s.d.; two-tailed Student's $t$ test.

Supplementary Fig. 6 Chmp5-deficiency causes mitochondrial dysfunction to induce cell senescence.

a Representative confocal images of TMRE showing accumulation of mitochondria in $C h m p 5^{\text {Ctsk }}$ skeletal progenitors. $n=30$ cells per group, scale bars, $25 \mu \mathrm{m}$. b Additional TEM images showing the morphology of mitochondria in $C h m p 5^{\text {Ctsk }}$ versus wild-type skeletal progenitors. $n=$ 50 cells per group; scale bars, $200 \mathrm{~nm}$.

Supplementary Fig. 7 X-ray images of $\mathrm{Chmp5}^{\mathrm{Ctsk}}$ mice after treatment with $\mathrm{Q}+\mathrm{D}$ or the vehicle PEG400 for 7 weeks. Arrows indicating bone overgrowths.

Supplementary Fig. 8 Eliminating senescent cells mitigates bone overgrowth and myopathy in $\operatorname{Chmp5}^{\text {Ctsk }}$ and $\operatorname{Chmp5}^{\mathrm{Dmp1}}$ mice a X-ray images of $C h m p 5^{f l f l}$ control mice after treatment with $\mathrm{Q}+\mathrm{D}$ or the vehicle PEG400 for 7 weeks. b Gross images showing hindlimb abduction in $C h m p 5^{D m p l}$ and $C h m p 5^{f l f l}$ mice after treatment with Q + D or the vehicle PEG400. $n=12$ animals per group.

Supplementary Table 1 Enriched gene lists by GSEA for cell senescence-related pathways Supplementary Table 2 Ingenuity Pathway Analysis - osteoblast differentiation-related pathways 


\section{a}

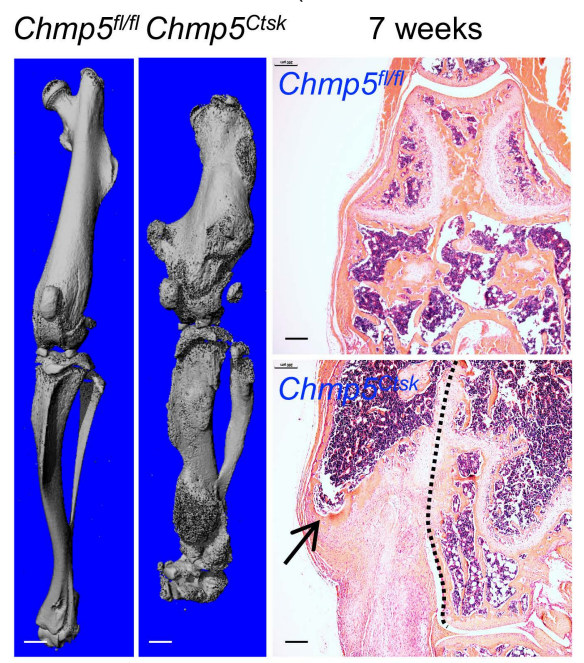

d

Chmp5 $5^{\text {Ctsk/+;Rosa26 }}{ }^{\text {mTmG/+ }}$
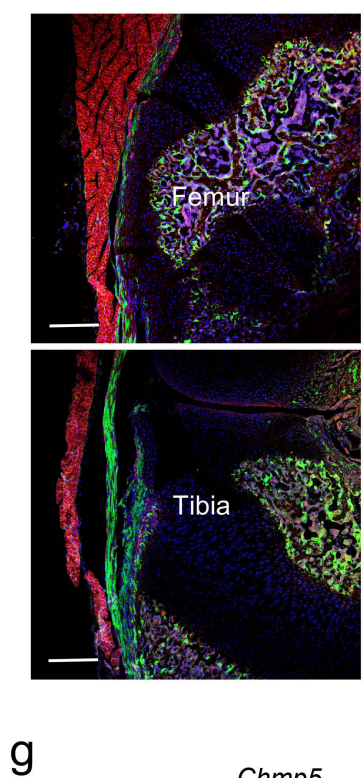

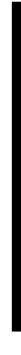

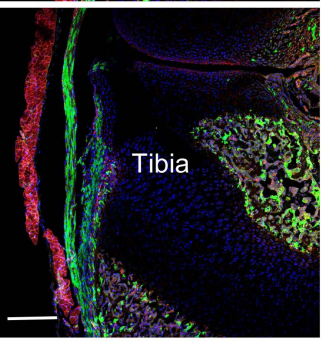

Chmp5

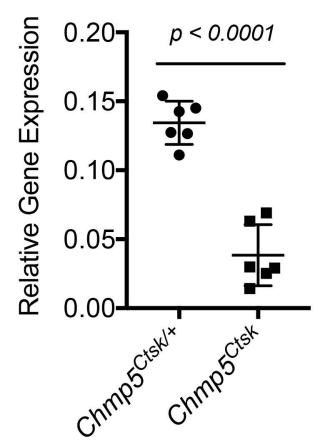

Chmp5 ${ }^{\text {Ctsk} ; R o s a 26 ~} 6^{m T m G /+}$
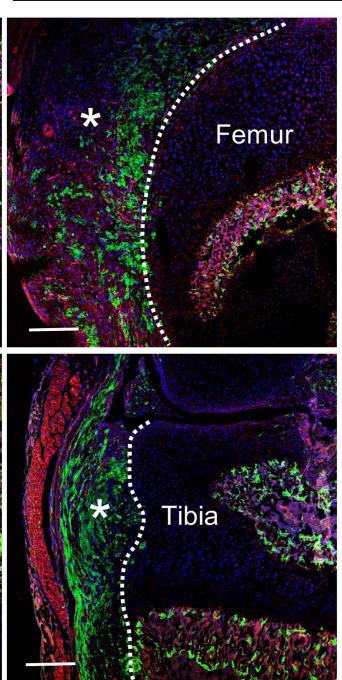
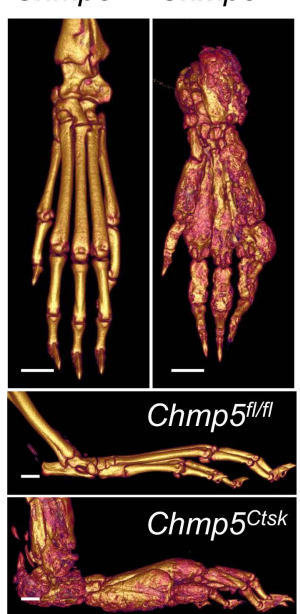
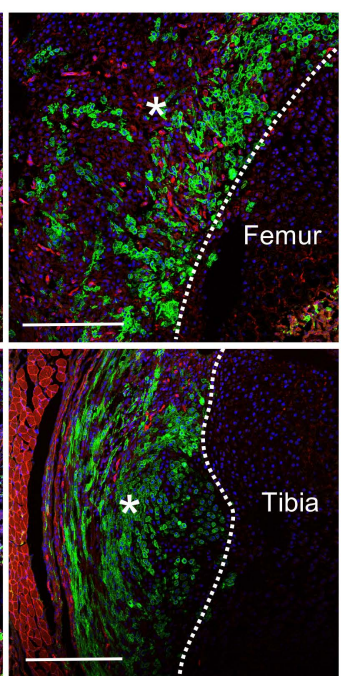

e
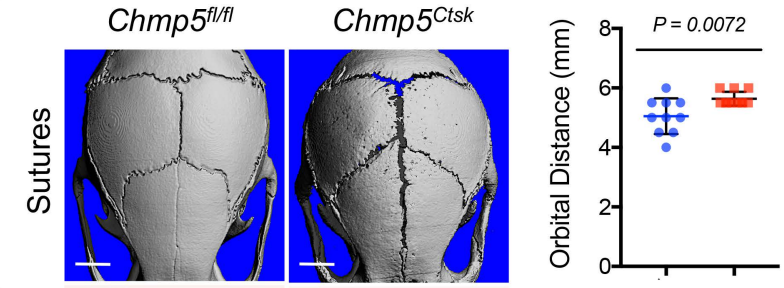

흔

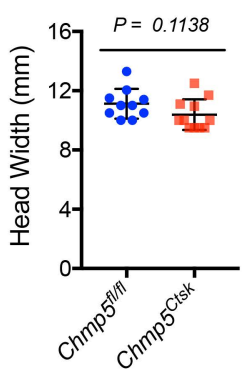

Chmp5 $5^{\text {Ctsk/+;Rosa26mTmG/+ } \quad \text { Chmp5 }}{ }^{\text {Ctsk;Rosa26mTmG/+ }}$
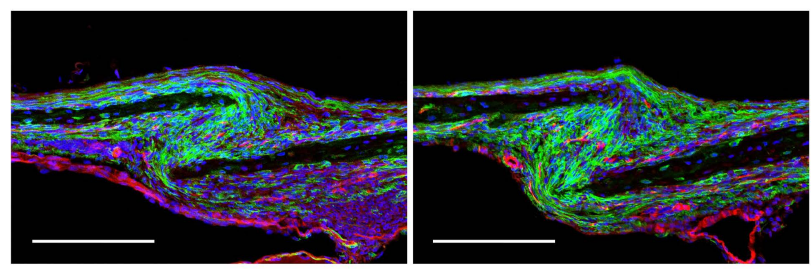

f

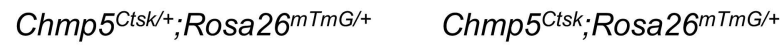
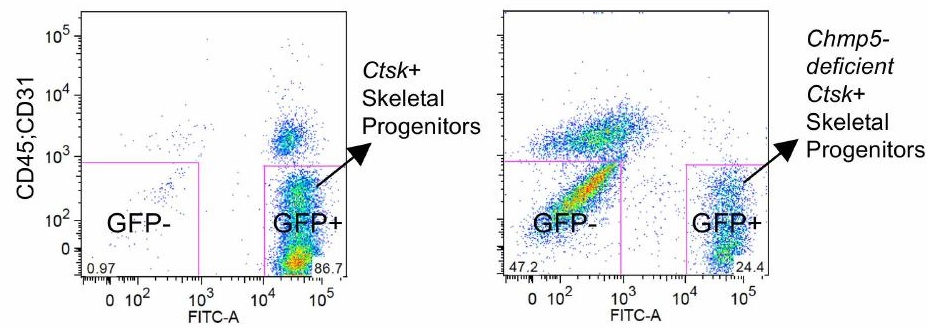

h
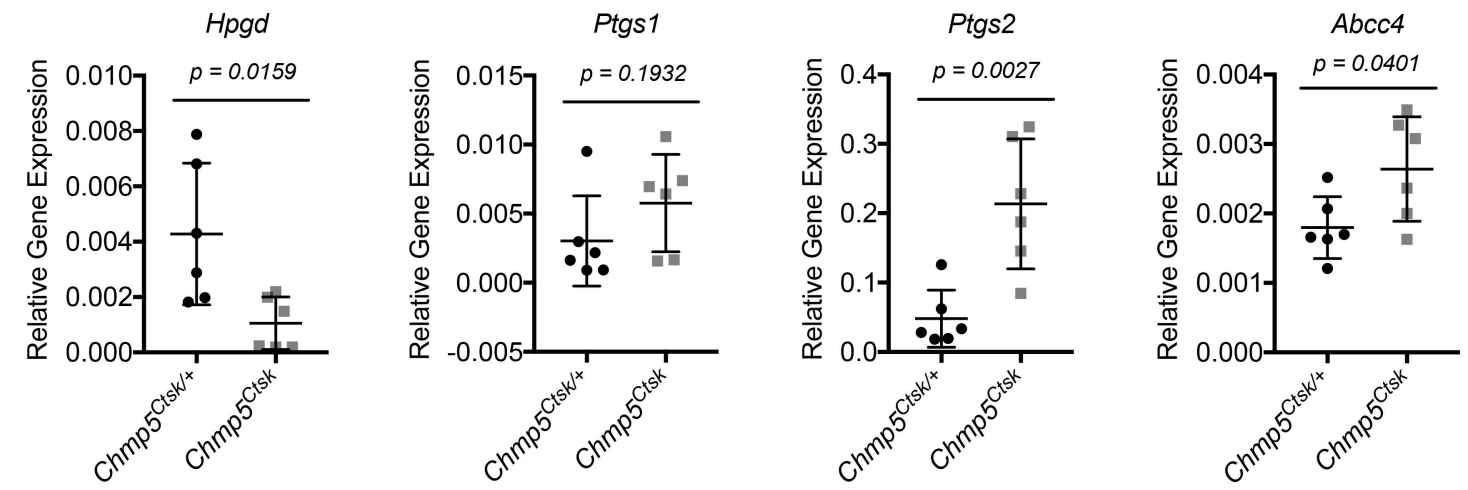

Fig. 1 
5 weeks

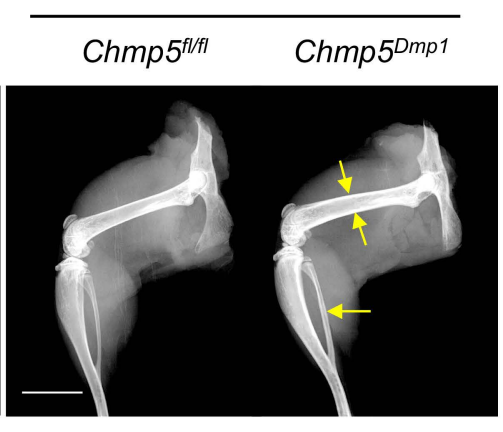

10 weeks

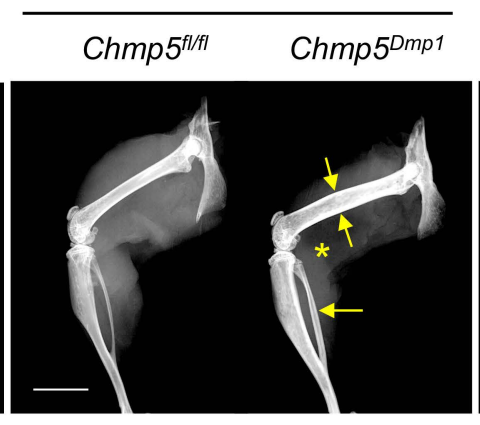

1 year

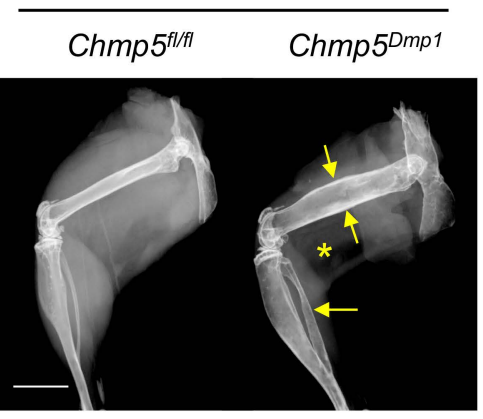

b

Chmp5 $5^{\text {fl/fl }}$ Chmp5 $5^{\mathrm{Dmp} 1}$

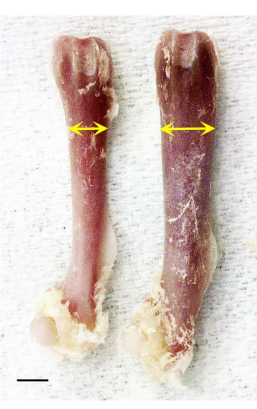

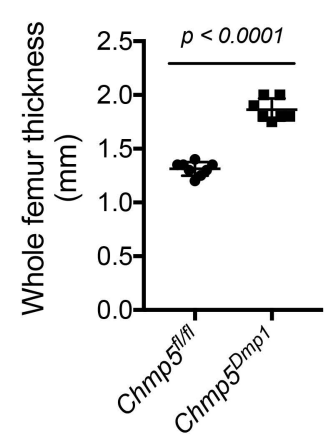

C

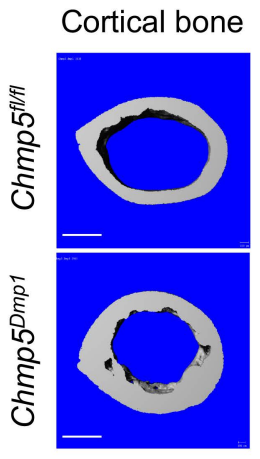

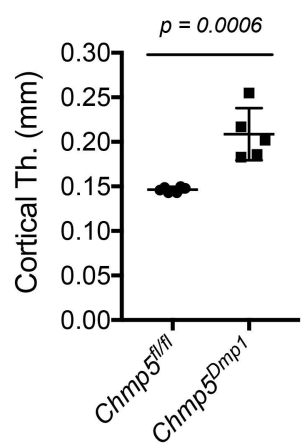

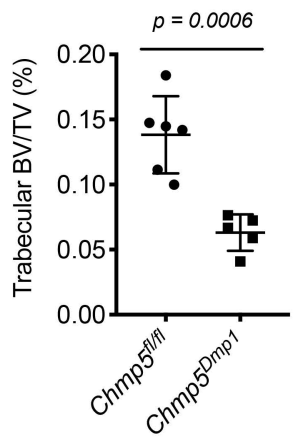

d
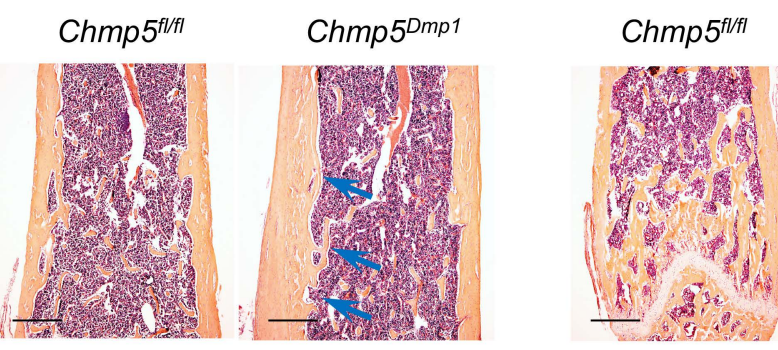

Chmp5 $5^{\mathrm{Dmp} 1}$

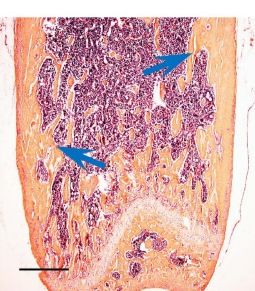

e

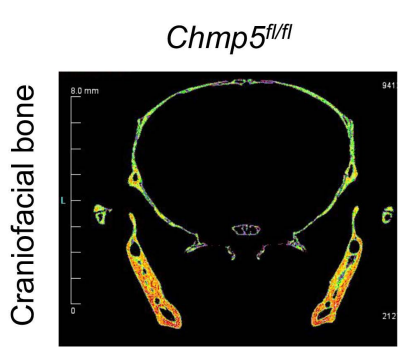

Chmp5 $5^{\text {Dmp } 1}$

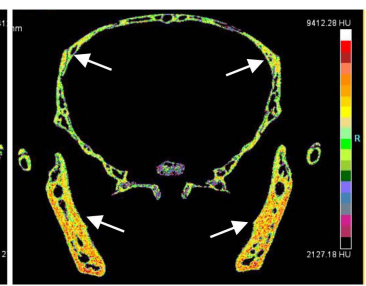

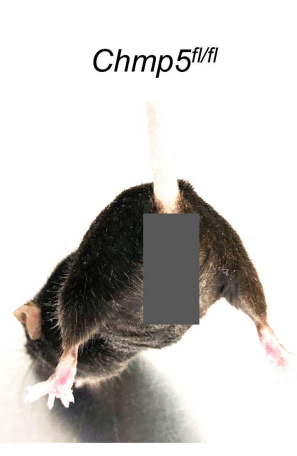

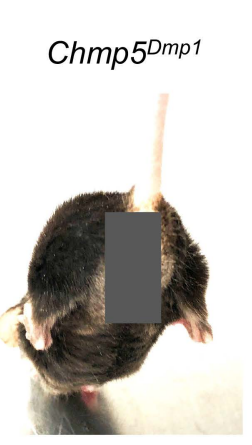

g

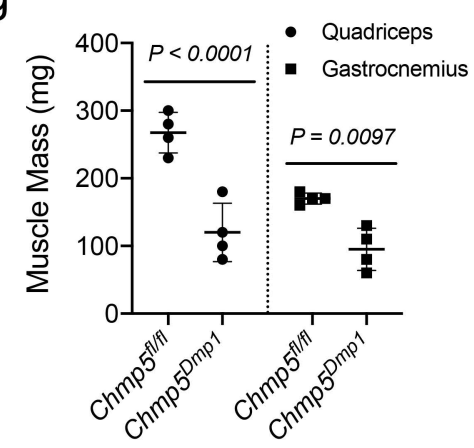

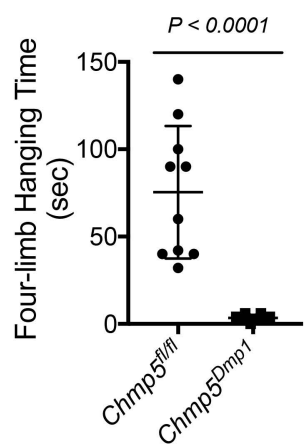

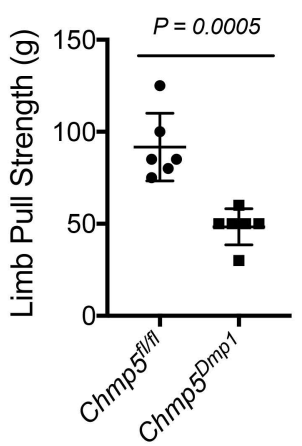

Fig. 2 
(which was not certified by peer review) is the author/funder. All rights reserved. No reuse allowed without permission.

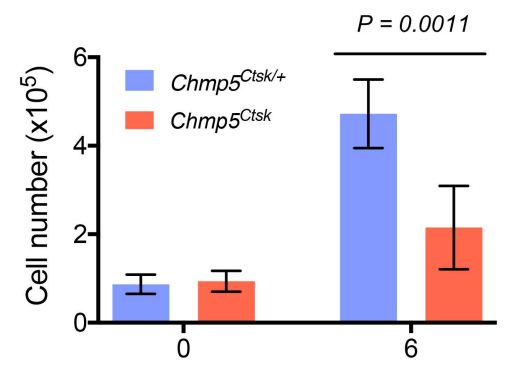

Days in culture
Two weeks of age

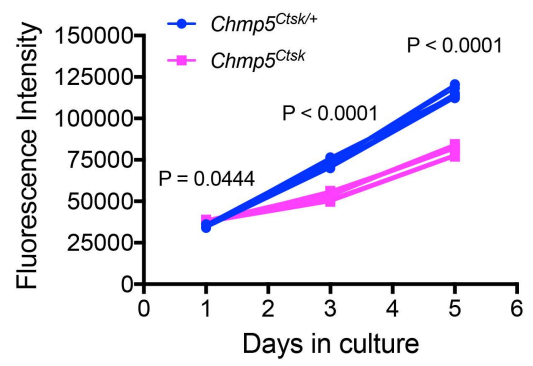

Cell Senescence

Chmp5 ${ }^{\text {Ctsk }}$ vs. Chmp5 Ctskt $^{+}$

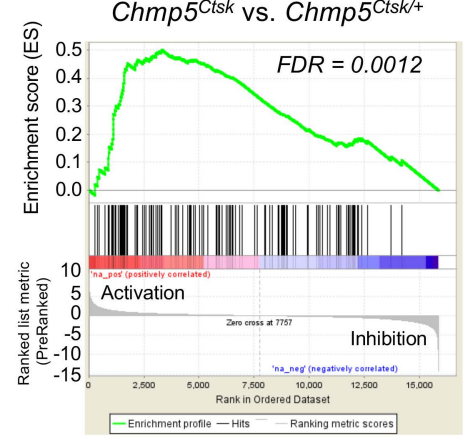

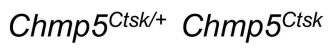

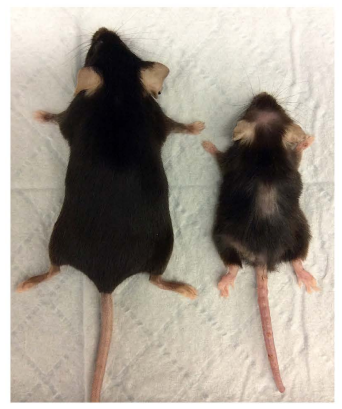

d

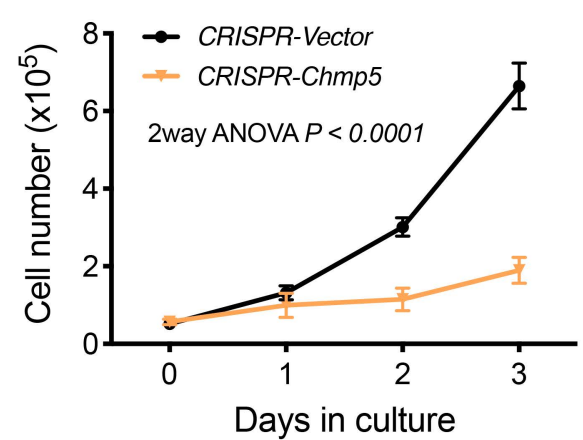

e
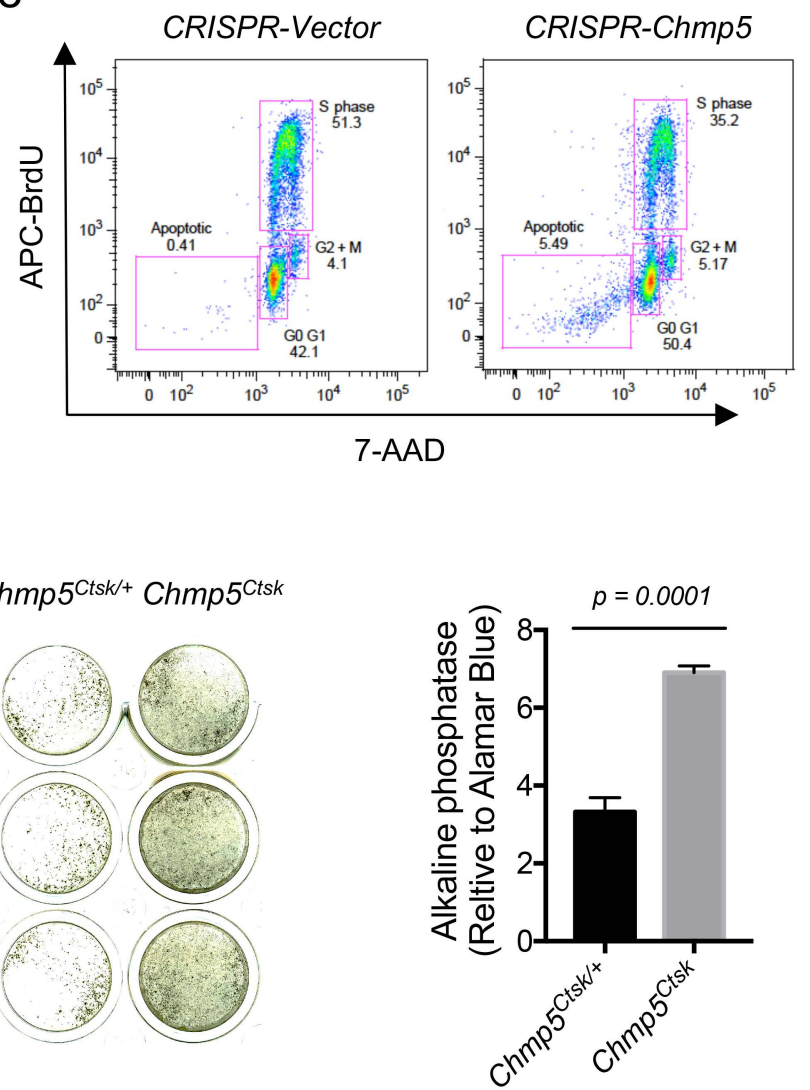

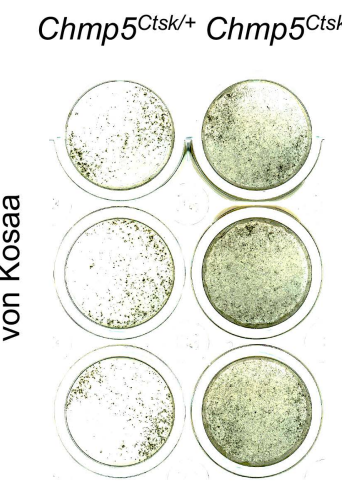

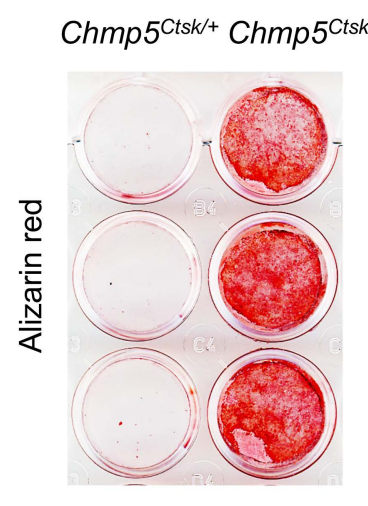

Ingenuity pathway analysis

\begin{tabular}{lcccc}
\hline \multicolumn{1}{c}{$\begin{array}{c}\text { Diseases or Functions } \\
\text { Annotation }\end{array}$} & $\mathrm{p}$-Value & $\begin{array}{c}\text { Predicted Activation } \\
\text { State }\end{array}$ & $\begin{array}{c}\text { Activation } \\
\text { z-score }\end{array}$ & \# Molecules \\
\hline $\begin{array}{l}\text { Differentiation of osteoblastic- } \\
\text { lineage cells }\end{array}$ & 0.00000101 & Increased & 2.365 & 44 \\
Differentiation of bone & $6.05 \mathrm{E}-11$ & Increased & 2.229 & 71 \\
Differentiation of osteoblasts & 0.00000197 & Increased & 2.101 & 43 \\
Differentiation of bone cells & $1.05 \mathrm{E}-10$ & Increased & 2.1 & 70 \\
\hline
\end{tabular}

$\mathrm{h}$
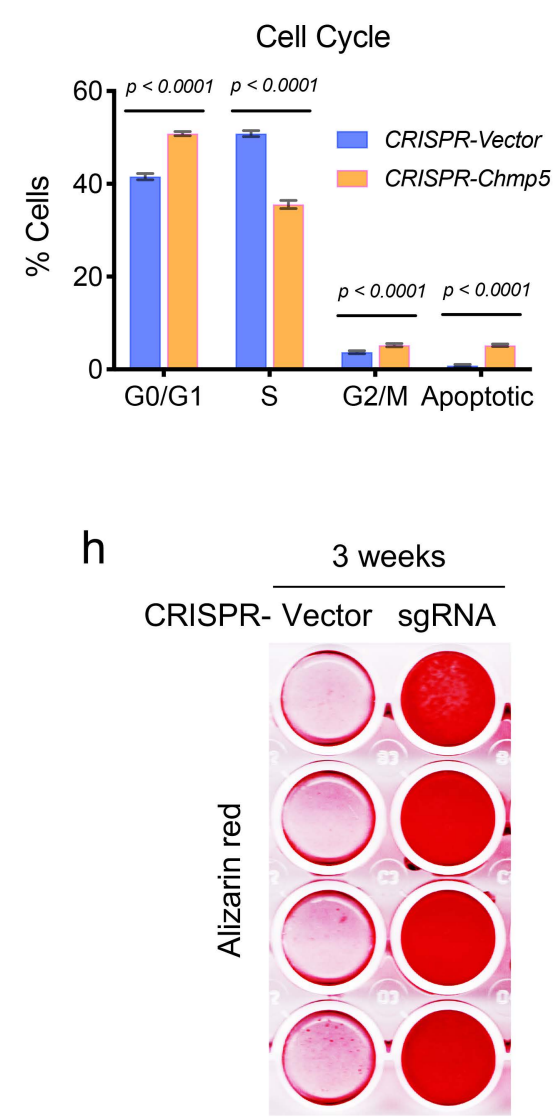

Fig. 3

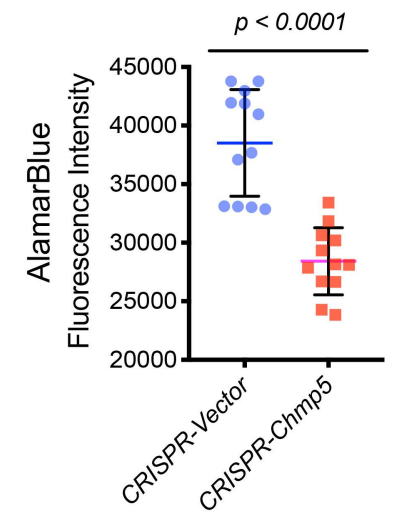


a (which was not certified by peer review) ib the author/funder. All rights reserved. No reuse allowed without permission.

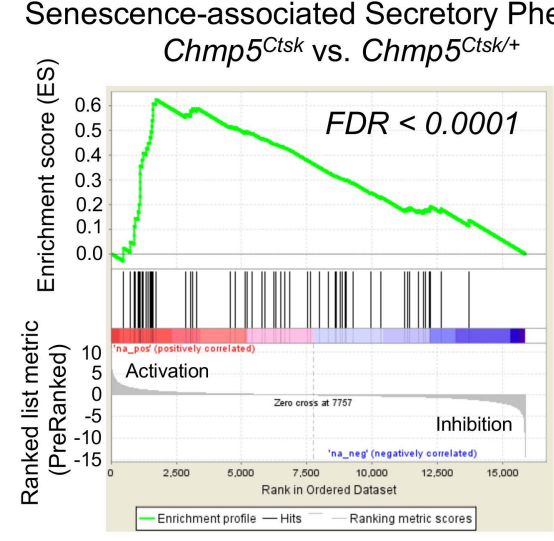

Wild-type

Chmp5 ${ }^{\text {Ctsk }}$

C

Plate same \# of cells and culture overnight

Wash and change to serum-free medium

$$
\downarrow \text { Culture for } 9 \mathrm{hrs}
$$

Collect medium Spin at $300 \mathrm{~g}, 10 \mathrm{~min}$

$$
\downarrow \text { Supernatant }
$$

Spin at $2000 \mathrm{~g}, 20 \mathrm{~min}$

$$
\downarrow \text { Supernatant }
$$

Spin at 10, $000 \mathrm{~g}, 30 \mathrm{~min}$

$$
\downarrow \text { Supernatant }
$$

NanoSight analysis d

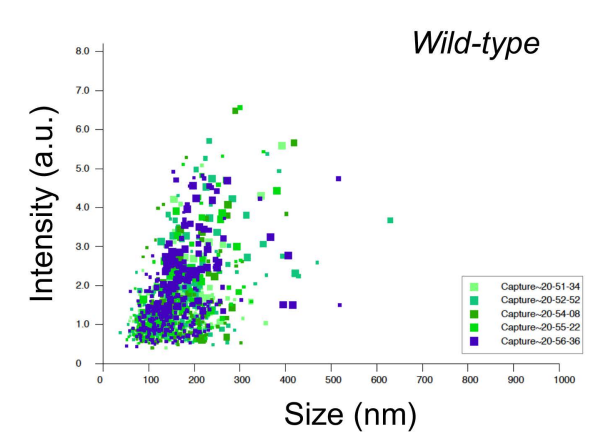

g

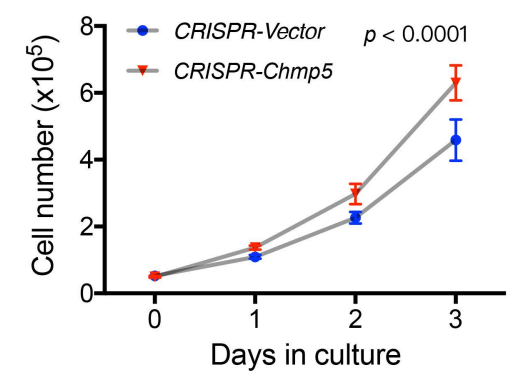

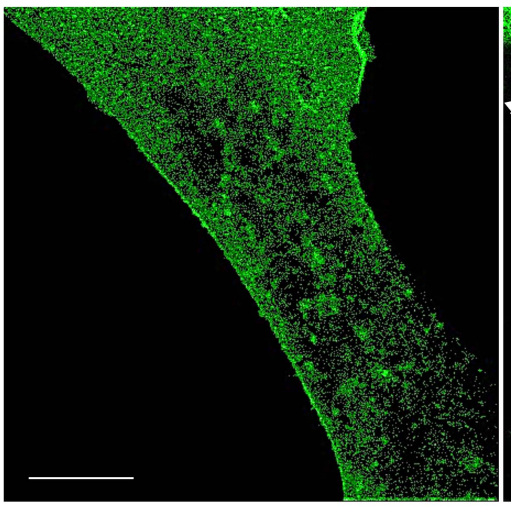

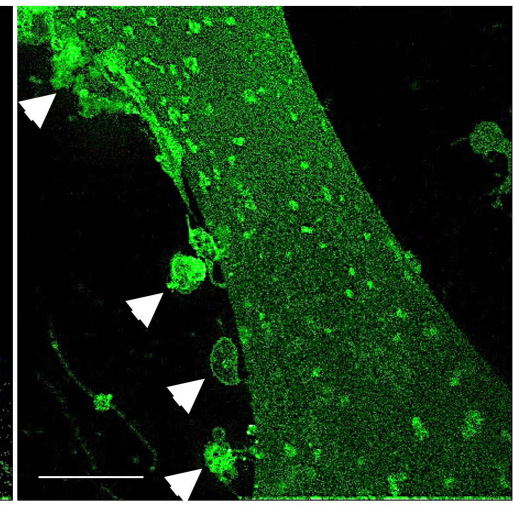

e
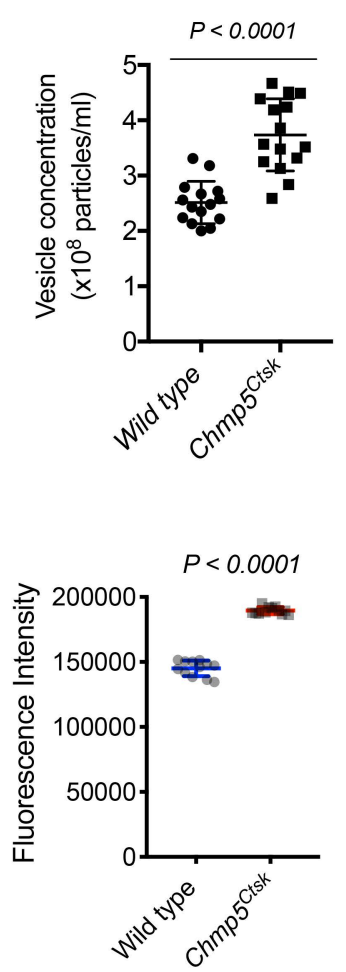

h

2 weeks

$\overline{\text { Wild-type Chmp5 }{ }^{\text {Ctsk }}} \overline{\text { Wild-type Chmp5 } 5^{\text {Ctsk }}}$

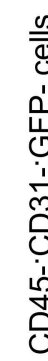

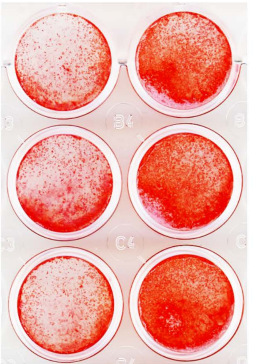

Chmp5 $5^{\text {Ctsk }+}+$

Chmp5 $5^{\text {Ctsk }}$

Ki-67 immunostaining

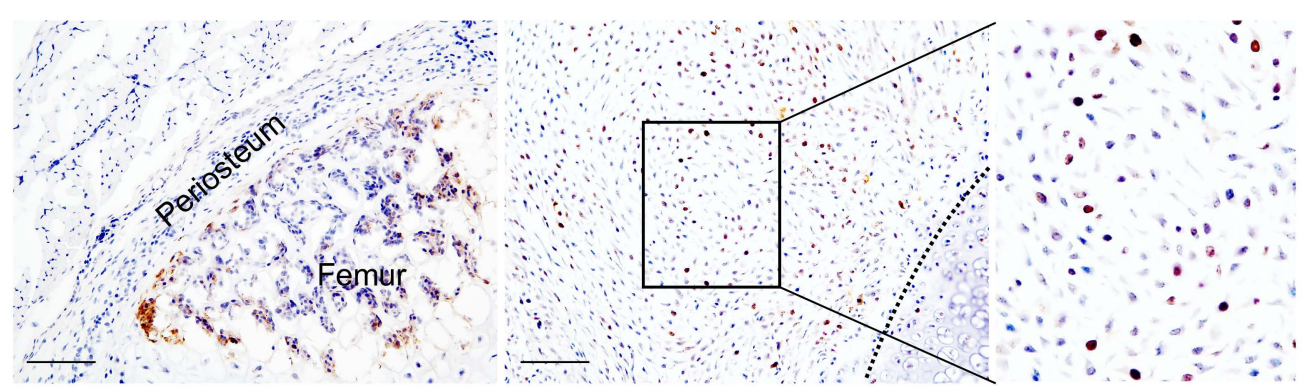

Fig. 4 
$a$

(which was not certified bjpeer review) is the author/funder. All rights reserved. No reuse allowed without permission.

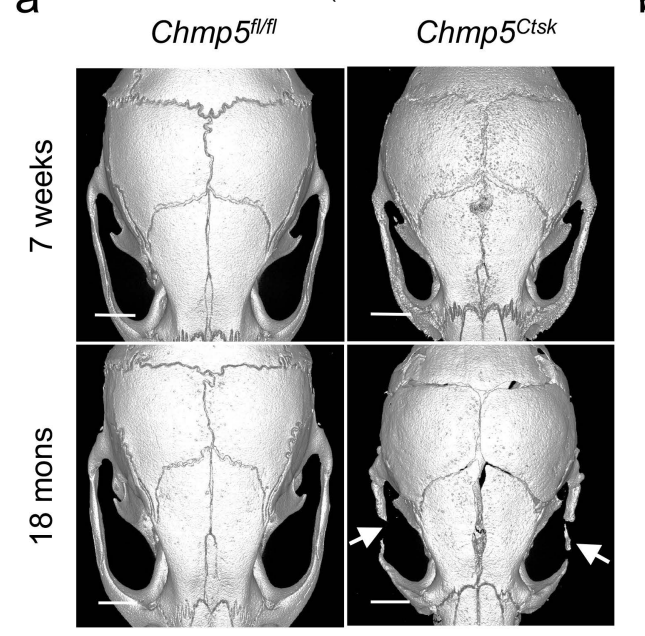

Chmp5 $5^{\text {Ctsk/+ }}$
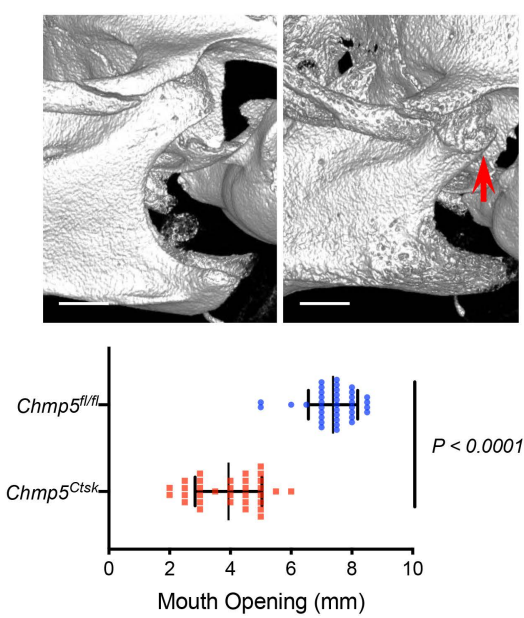

Chmp5 $5^{\text {Ctsk }}$

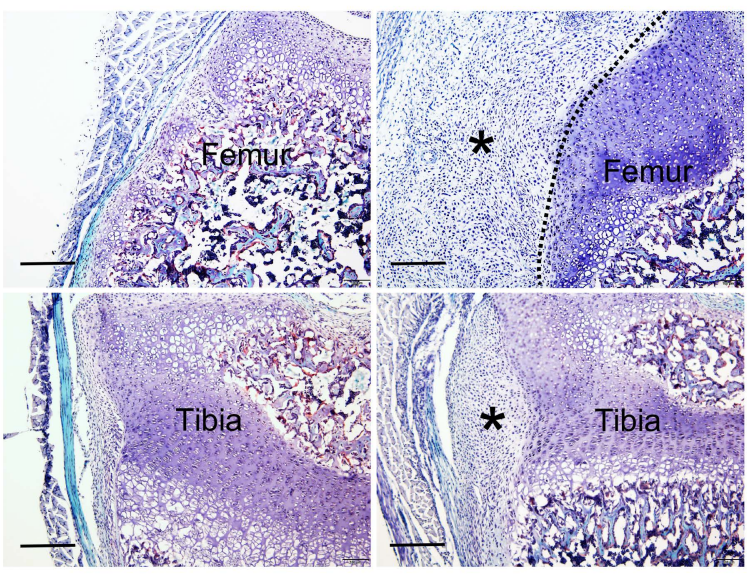

e

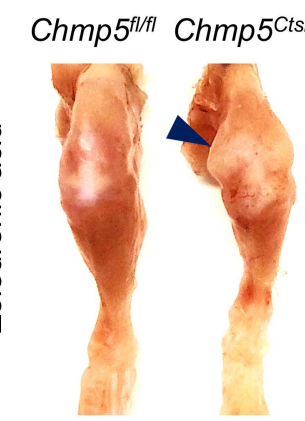

Chmp5 $5^{\text {Ctsk }}$
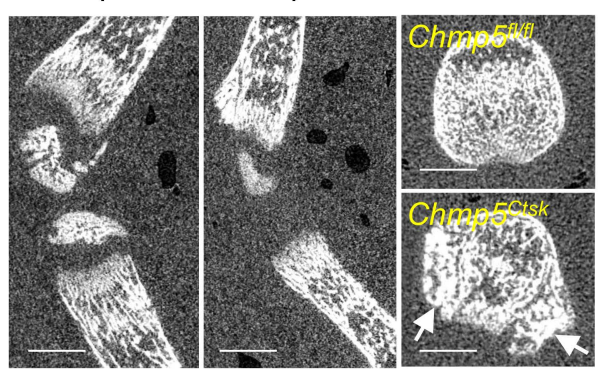

f

Chmp5 $5^{\text {flfl }}$ Chmp5 $5^{\text {Ctsk }}$ Chmp5 $5^{f / f l} \quad$ Chmp5 $5^{\text {Ctsk }}$

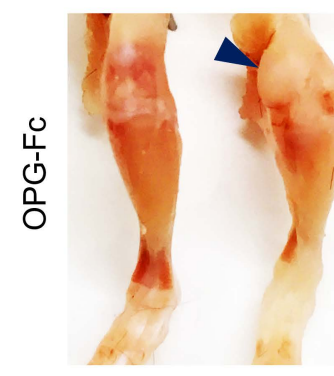

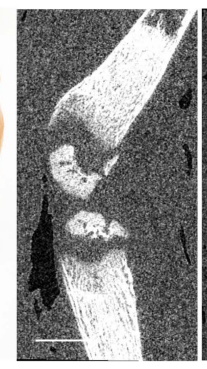

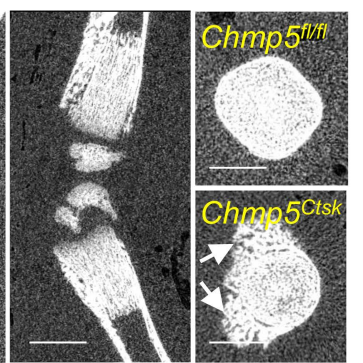

g

Chmp5 $5^{\text {flffl }} \quad$ Chmp5 $^{\text {Ctsk }}$ Chmp5 $5^{\text {flftl }}$ Chmp5 $5^{\text {Ctsk }}$

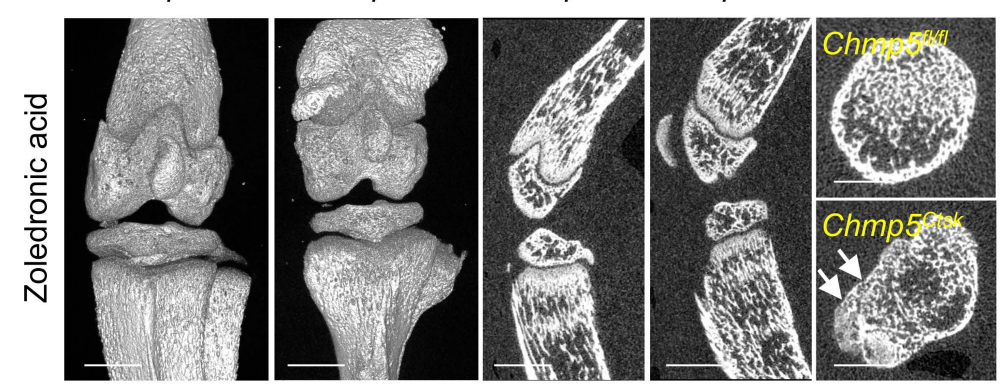

$\mathrm{h}$ Chmp5 $5^{f / f l}$

Chmp5 $5^{\text {Ctsk }}$

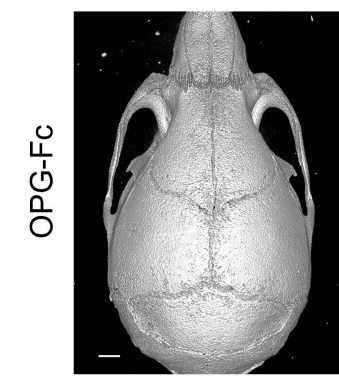

Cre negative control

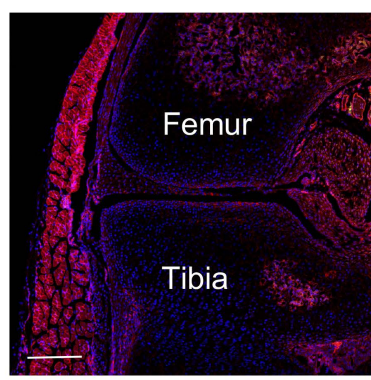

Cre negative control

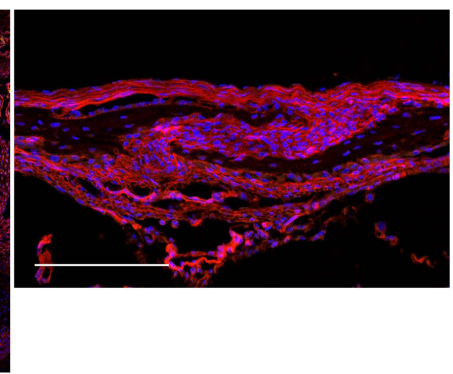

Cre negative control

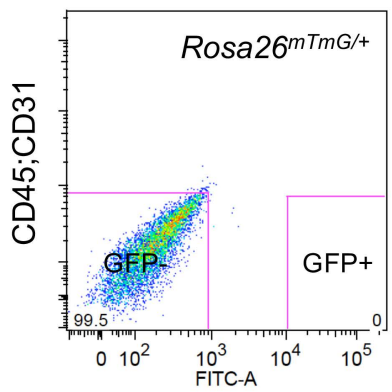

Supplementary Fig. 1 
a (which was not certified by peer review) is the author/funder. All rights reserved. No reuse allowed without permission.

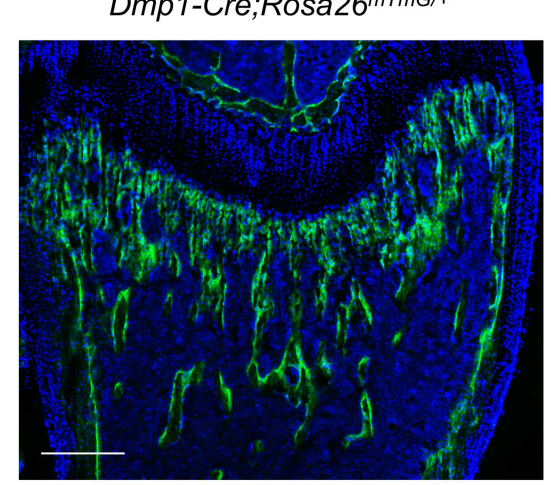

C
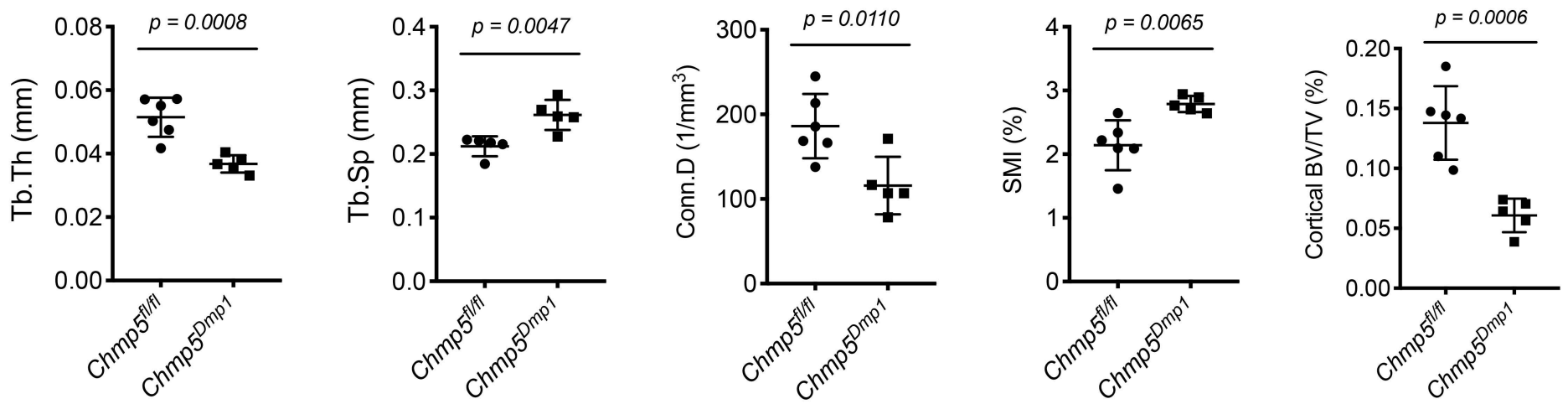

d

Chmp5 $5^{\text {Dmp 1/+ }}$

Chmp5 $5^{\text {Dmp1 }}$

M-CSF
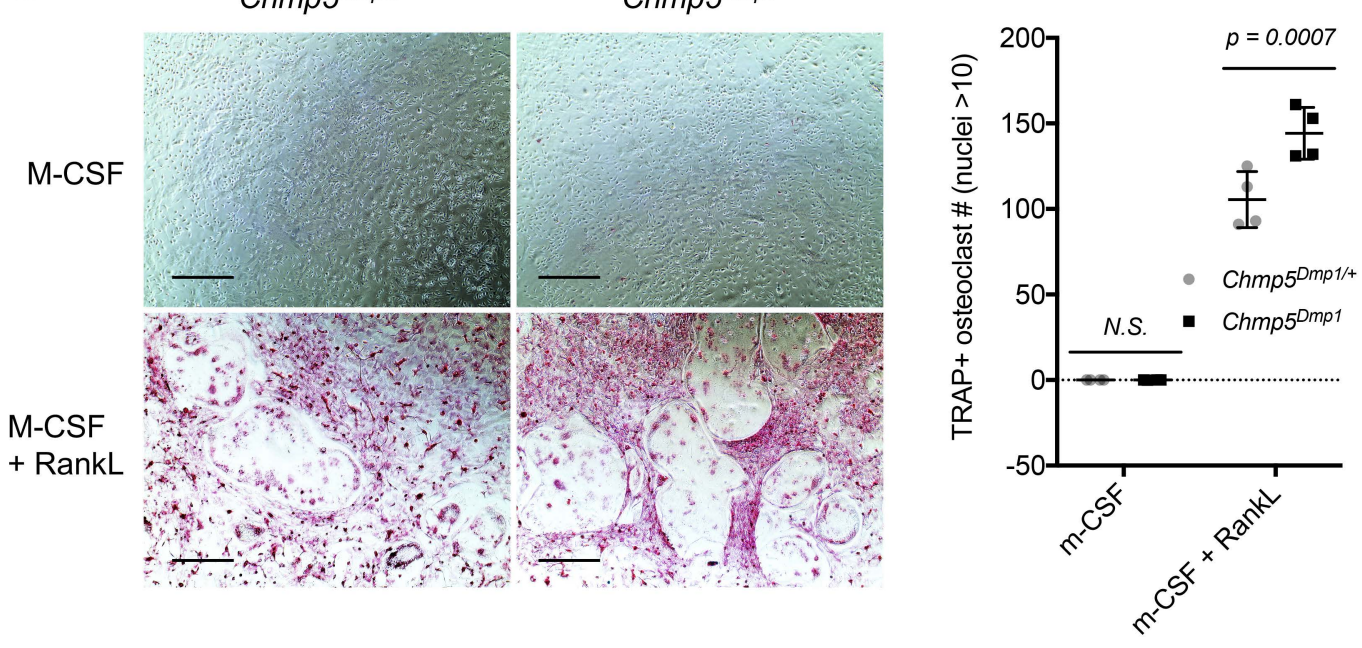

TRAP activity in supernatants
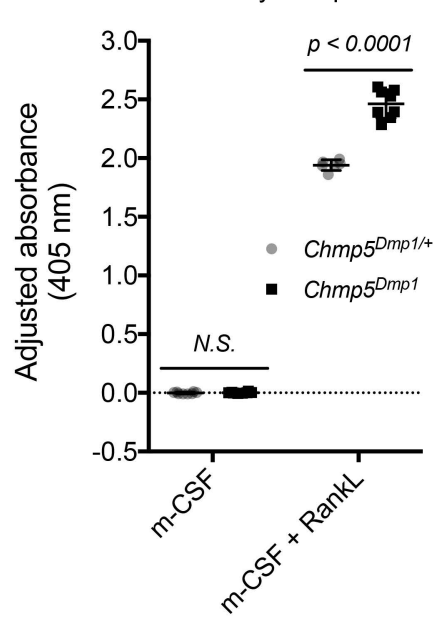

Supplementary Fig. 2
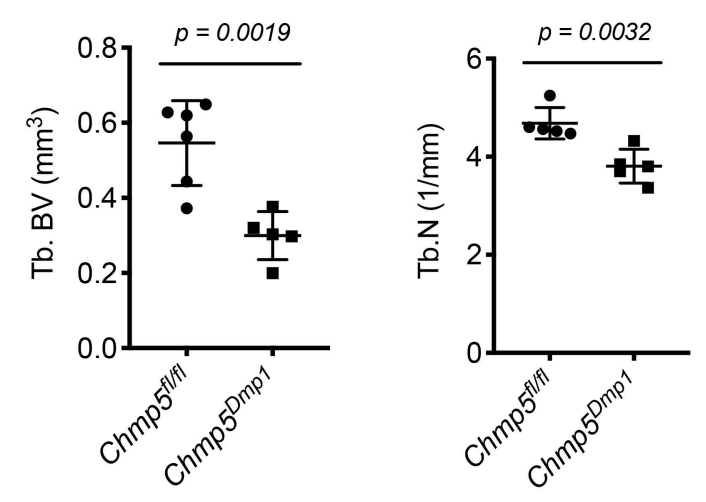

+ RankL

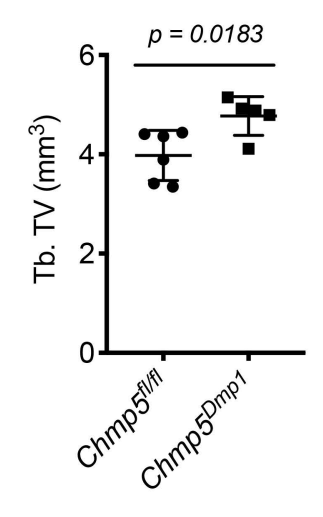




\section{a}

(which was not certified by peer review) is theauthor/funder. All rights reserved. No reuse allowed without permission.
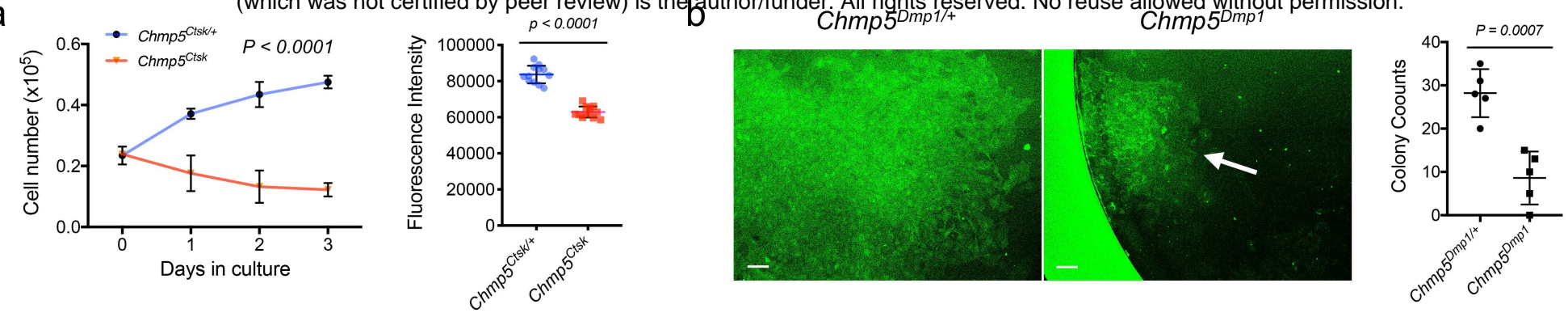

C
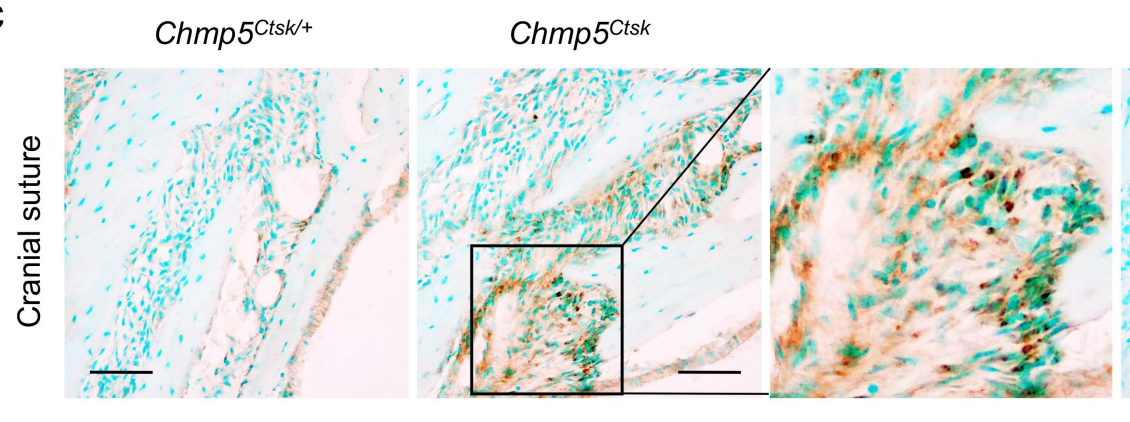

d

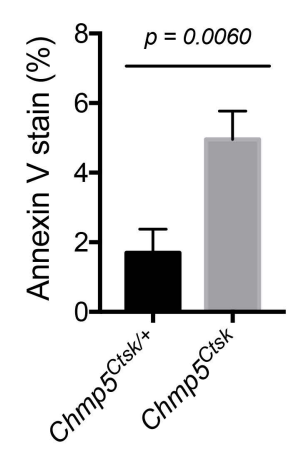

$\mathrm{h}$

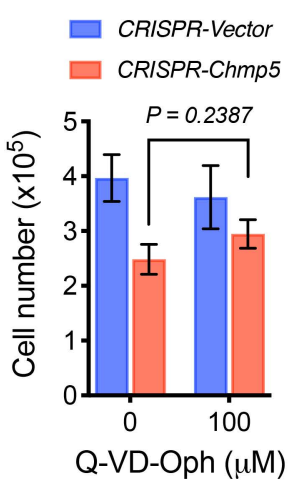

e

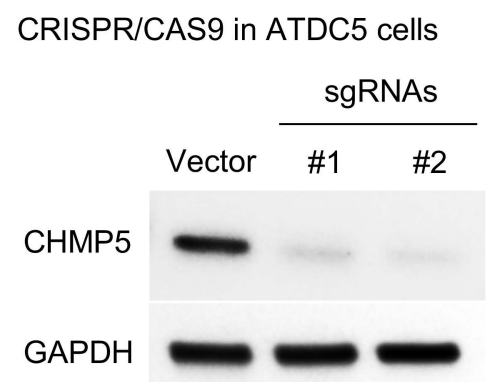

f

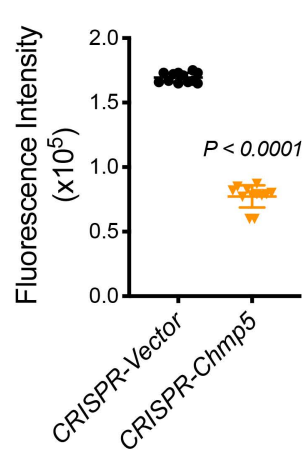

i
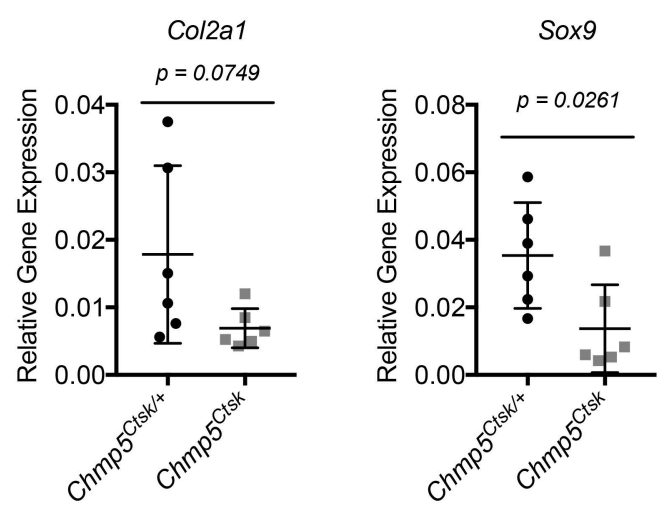

Negative control
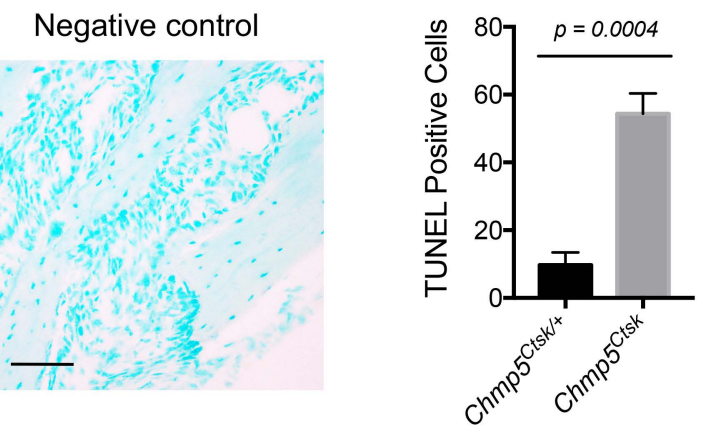

g
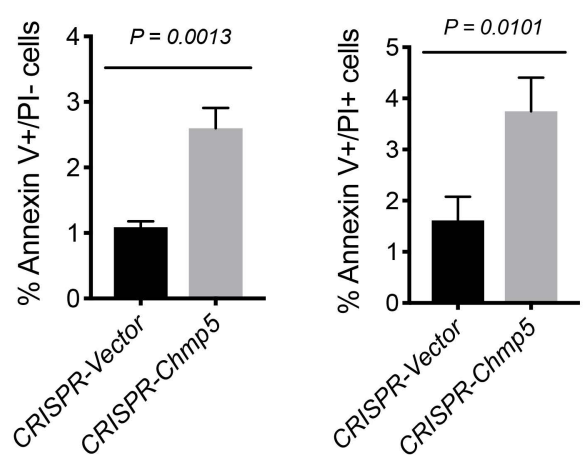

k

CRISPR/CAS9 in MC3T3-E1 cells

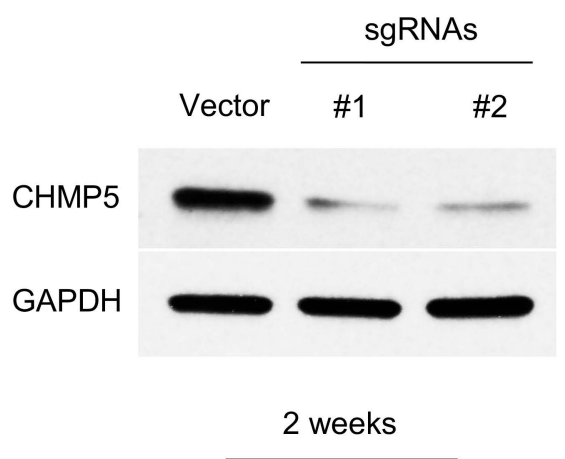

CRISPR- Vector sgRNA

PANTHER GO-BP gene enrichment analysis

\begin{tabular}{lccc}
\hline GO biological process complete & number & OverUnder & P-value \\
\hline cartilage development (GO:0051216) & 137 & - & $2.13 \mathrm{E}-06$ \\
$\begin{array}{l}\text { endochondral bone morphogenesis (GO:0060350) } \\
\begin{array}{l}\text { cartilage development involved in endochondral } \\
\text { bone morphogenesis (GO:0060351) }\end{array}\end{array}$ & 51 & - & $1.06 \mathrm{E}-03$ \\
\begin{tabular}{l} 
chondrocyte differentiation (GO:0002062) \\
\hline
\end{tabular} & 61 & - & $6.62 \mathrm{E}-03$ \\
\hline
\end{tabular}

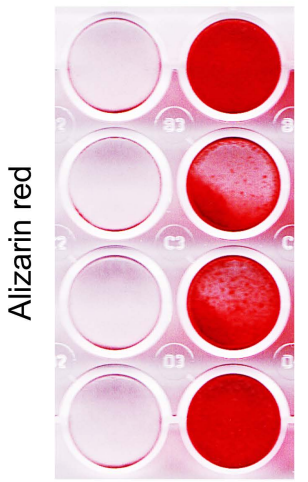


$a$ (which was n $\mathrm{BJ}$ certified by peer review) is the author/funder. All rights reserved. No reuse allowed without permission.
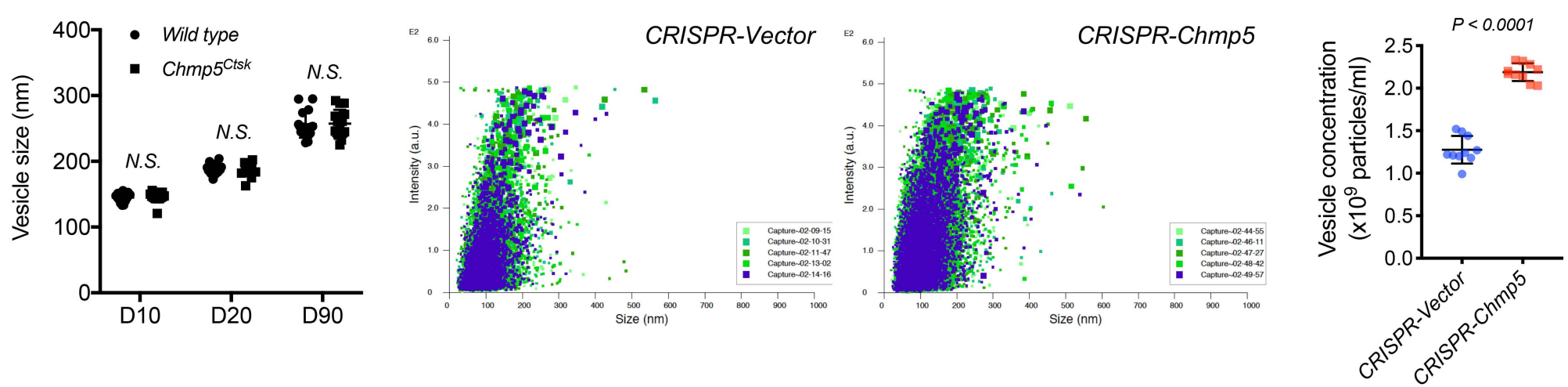

d

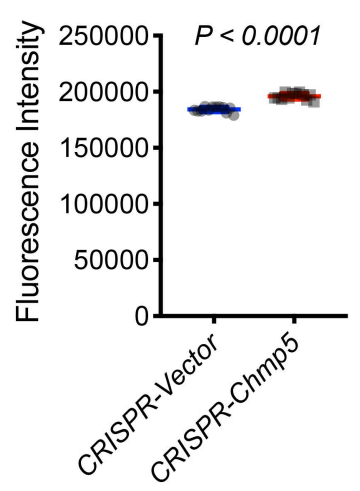

e

Chmp5 $5^{\text {Ctsk }++}$

Chmp5 $5^{\text {Ctsk }}$

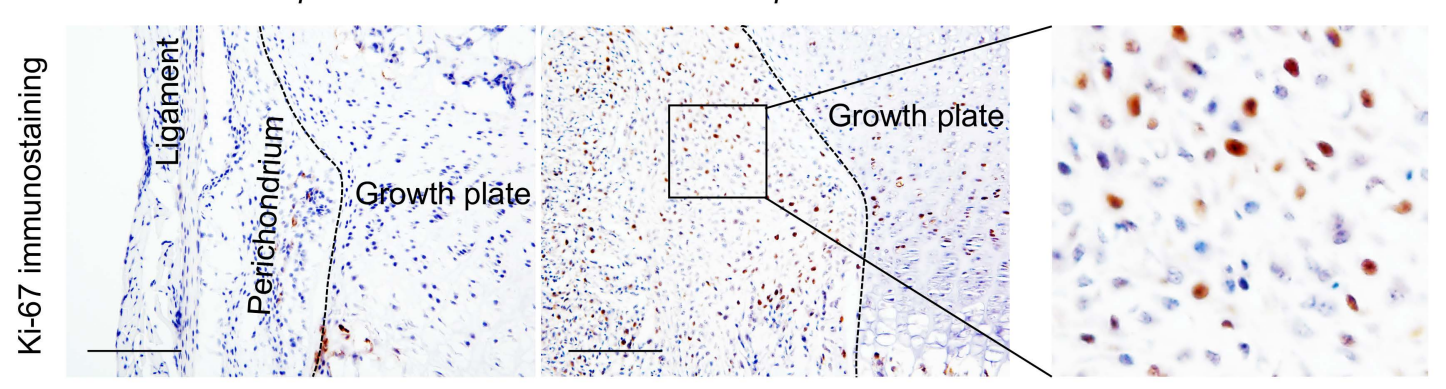

Upregulated genes coding secretory factors

Fold change $>1.5$ (Log2(fold change) $>0.585$ )

Adjusted $P<0.05$

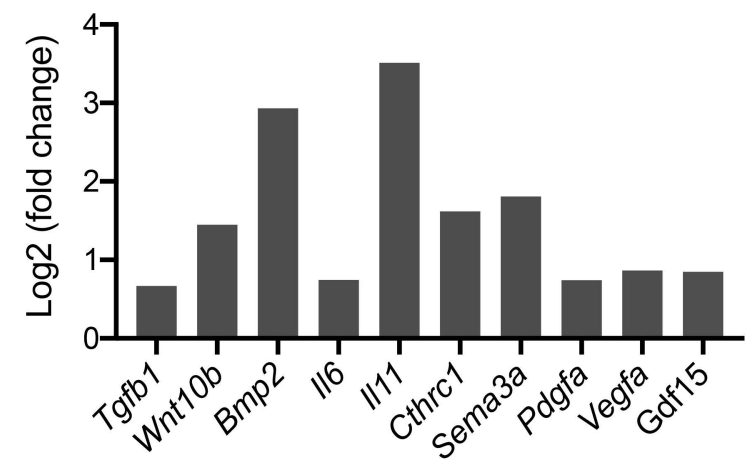

Supplementary Fig. 4 

(which was not certified by peer review) is the author/funder. All rights reserved. No reuse allowed without permission.

a

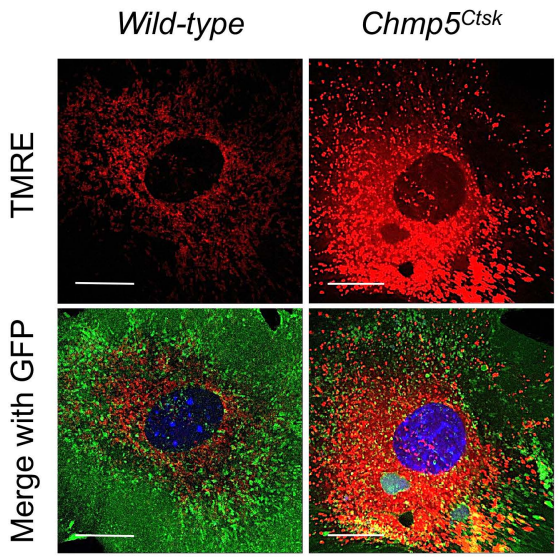

b

Wild-type

Chmp5 $5^{\text {Ctsk }}$

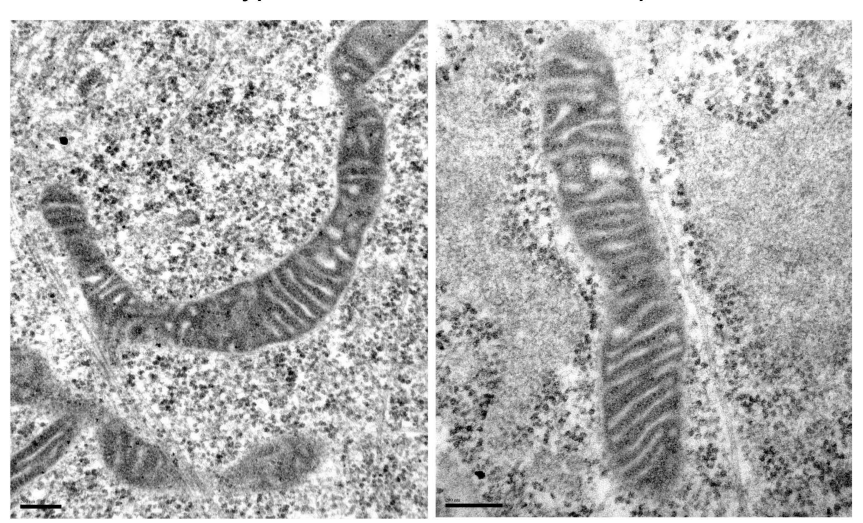

Supplementary Fig. 6 


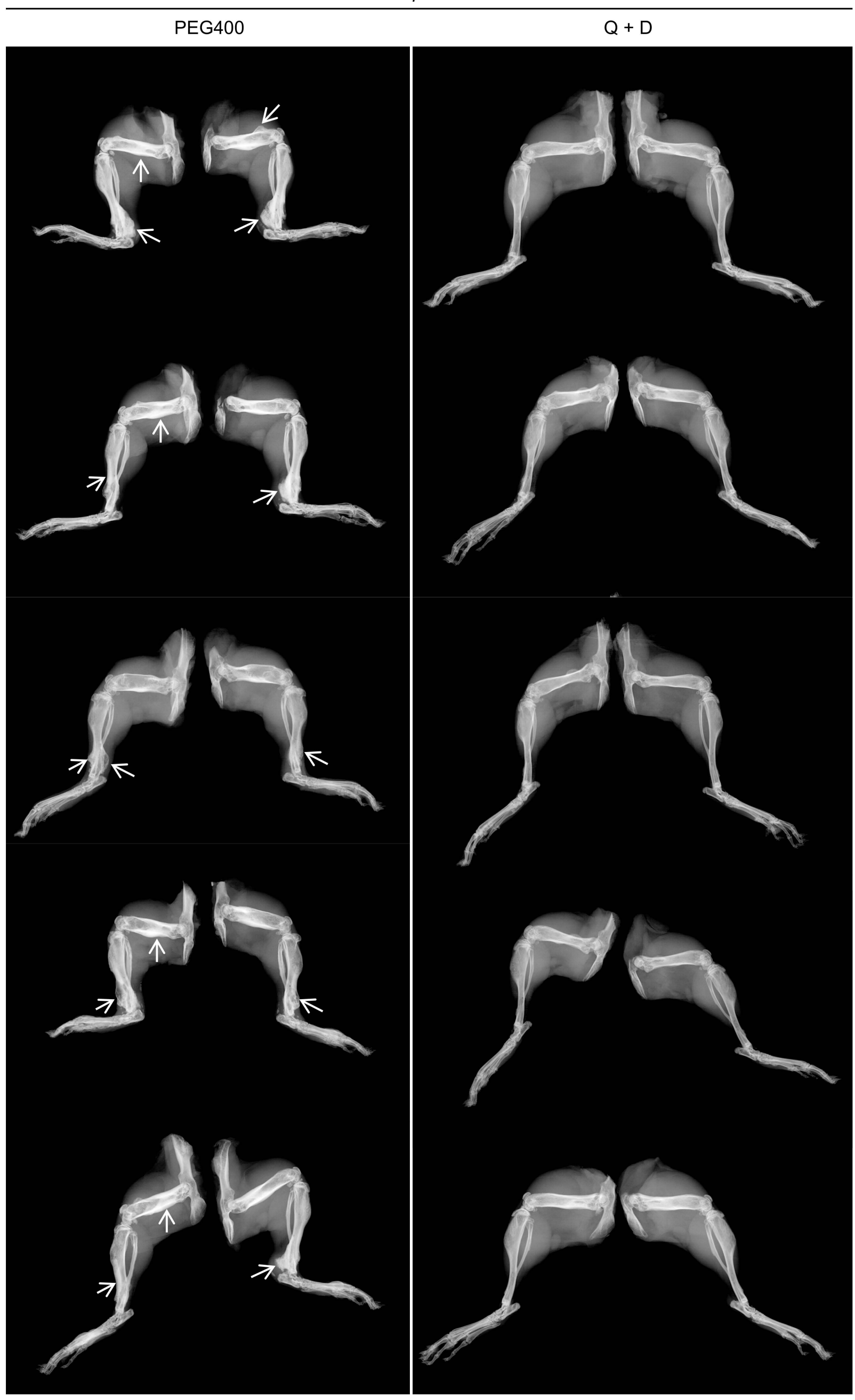

Supplementary Fig. 7 


PEG400 Q Q D
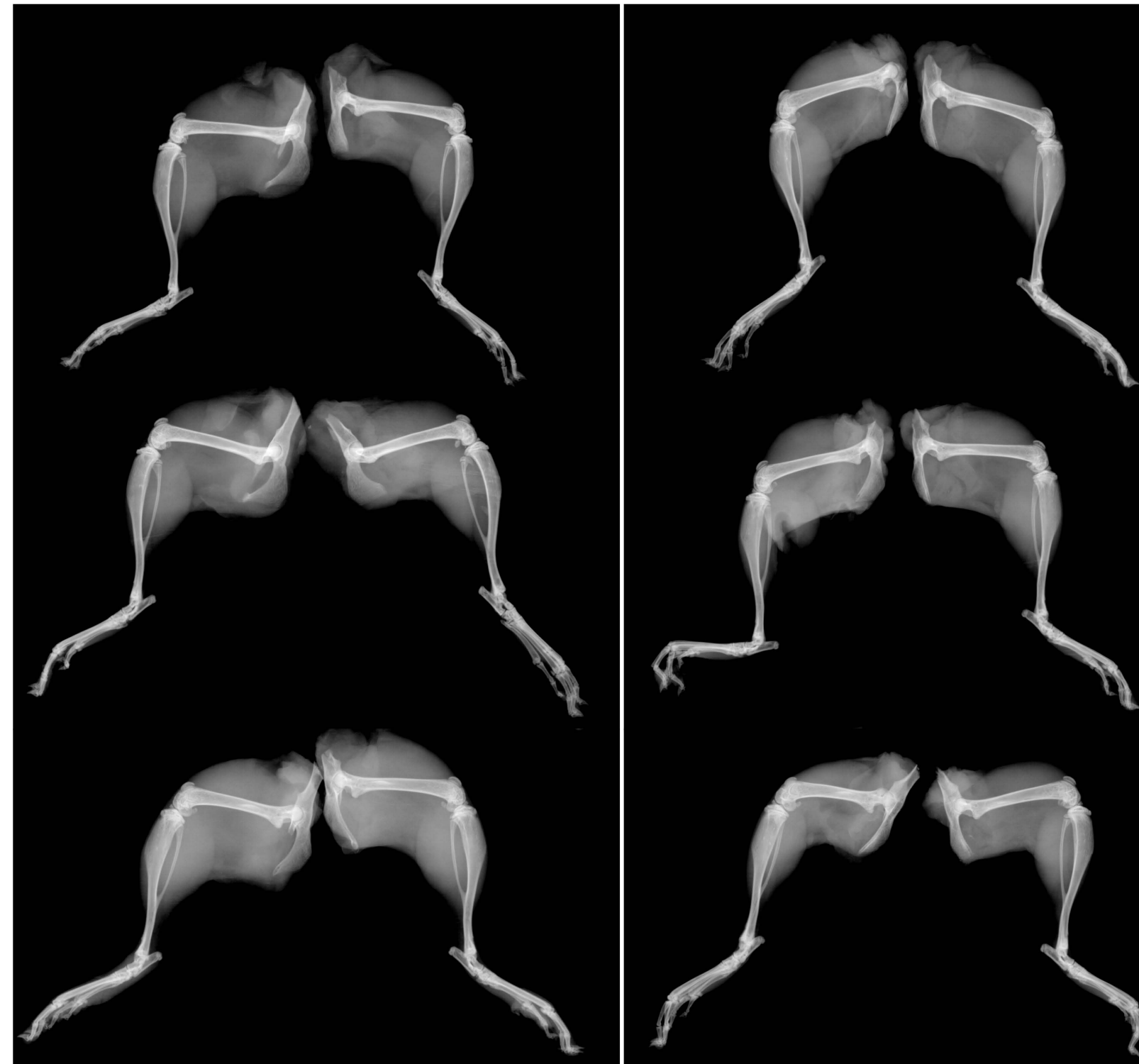

b

PEG400

$Q+D$

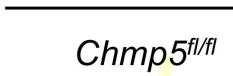

Chmp5 $5^{\text {Dmp } 1}$
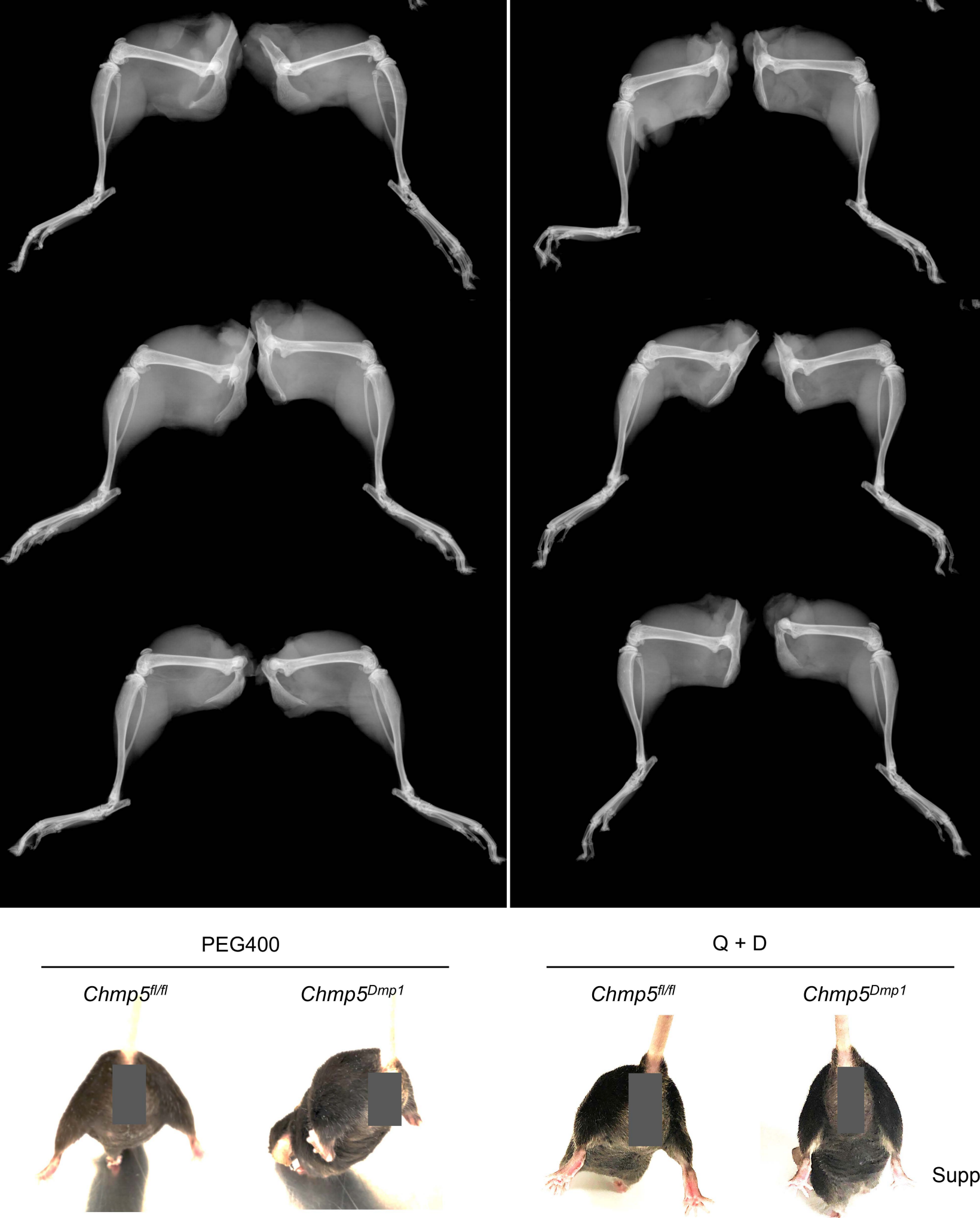

Chmp5 $5^{\text {Dmp1 }}$

Supplementary Fig. 8 